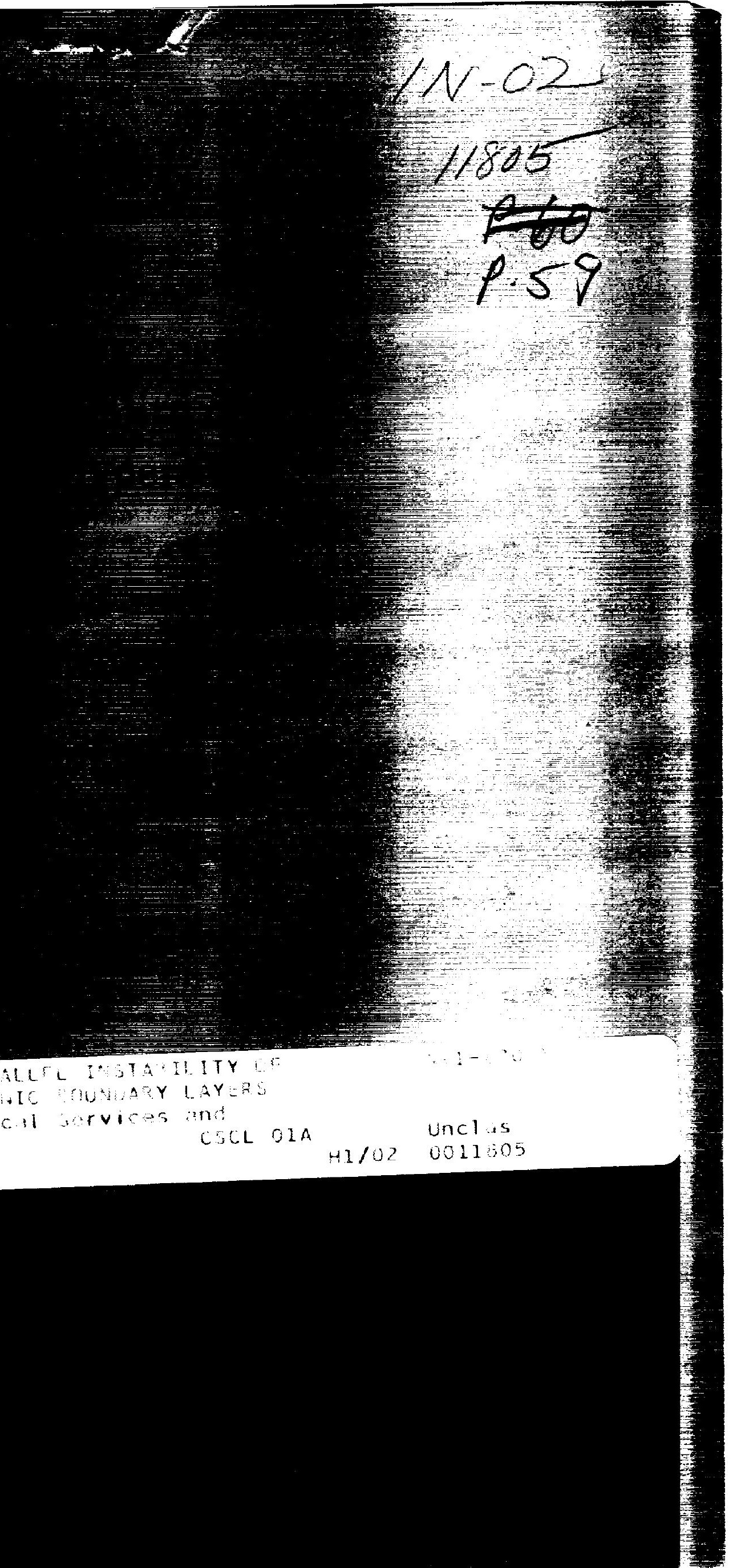




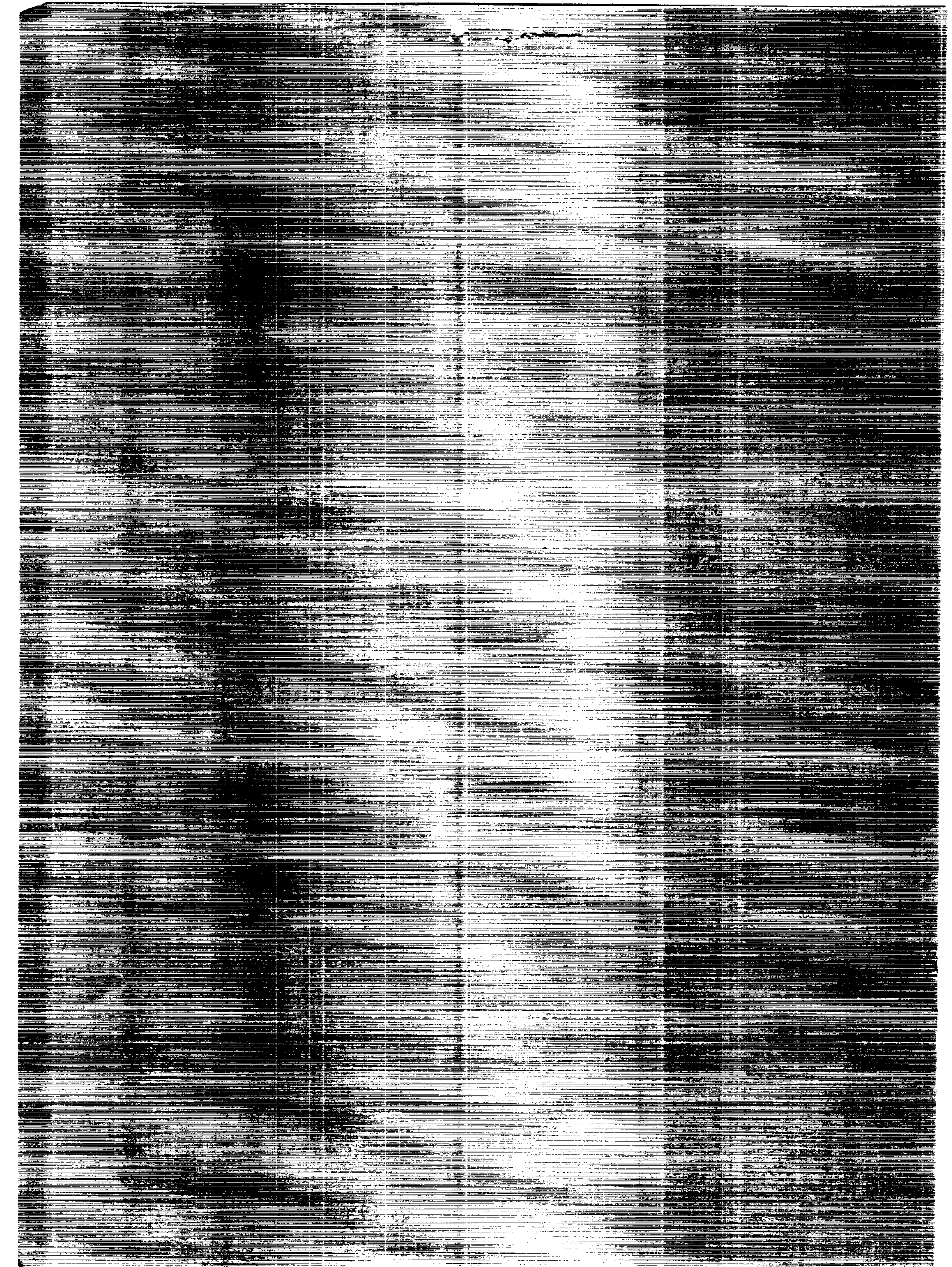


NASA Contractor Report 4373

\section{Nonparallel Instability of Supersonic and Hypersonic Boundary Layers}

Nabil M. El-Hady

Analytical Services \& Materials, Inc.

Hampton, Virginia

Prepared for

Langley Research Center

under Contract NAS1-18599

\section{NMSA}

National Aeronautics and

Space Administration

Office of Management

Scientific and Technical

Information Division 



\title{
NONPARALLEL INSTABILITY OF SUPERSONIC AND HYPERSONIC BOUNDARY LAYERS
}

\author{
Nabil M. El-Hady $\dagger$ \\ Analytical Services and Materials, Inc. \\ Hampton, Virginia 23666
}

\section{INTRODUCTION}

It is customary to use the parallel-flow approximation to describe the linear instability of boundary-layer flows to small amplitude disturbances. In spite of the qualitative success of this assumption, the parallel stability theory does not explain some important phenomena, and the experiments [1-3] have shown systematic differences with the theory.

Apart from predicting a minimum critical Reynolds number that is lower than that given by the parallel stability theory, evidence from experiments shows that the growth rate of the disturbance is not only a function of the coordinate normal to the wall, but also is different for different flow quantities. These phenomena occur due to the increase in the boundary-layer thickness. In cases where the mean boundary-layer flow changes rapidly due to localized adjustments, the parallel flow assumption is expected to fail, and the stability characteristics may not be accurately predicted. Also at the early stages of the nonlinear interactions, ElHady [4] has shown that nonparallel flow effects may control the initial development of the triad components in a triad resonant interaction model.

The comparison of the minimum critical Reynolds number between theory and experiment is always poor (this comparison exists only for incompressible flows). The large discrepancy occurs for high-frequency disturbances that exhibit weak amplification, and has often been attributed to nonparallel effects of the mean flow. Thorough analytical and Navier-Stokes investigations for incompressible flows [5] have confirmed that the nonparallel mean-flow 
effects, if accurately calculated, are weak for two-dimensional (2D) disturbances and consequently do not explain the discrepancy between the theory and the experiment at incompressible speeds. Some plausible reasons due to difficulties encountered in low speed experiments are discussed by Saric [6], which may have large effects on the experimental measurements specially for high frequency disturbances. Bertolotti [5] has conducted a sensitivity study on the neutral curves of $2 \mathrm{D}$ disturbances in incompressible flows. He investigated the effect of the distance from the wall at which the measurements are made, the effect of the presence of a slight adverse pressure gradient, the effect of the extent of transient response ( from a vibrating ribbon) following altered initial conditions, and the effect of nonlinear disturbance-amplitude levels used in the experiments. He found that all factors may contribute to the well known theoretical-experimental discrepancy at incompressible speeds.

The effect of the mean-flow nonparallelism on the stability and transition prediction of boundary layers is better illustrated by investigating the behavior of the growth rates of given disturbance frequency rather than by investigating its effect on the critical Reynolds number. For the flat plate compressible boundary layer, the boundary-layer thickness $\delta$ grows proportional to $M_{\infty}^{2}$, where $M_{\infty}$ is the free stream Mach number. Clearly, as the Mach number increases, the boundary layer becomes thicker and therefore the effect of the flow nonparallelism on the stability characteristics could be more pronounced for supersonic and hypersonic flows. For these speed ranges, accurate prediction of transition and transition control may be extremely beneficial, since skin friction and subsequent aerodynamic heating are considerably higher for turbulent flows at high speeds.

High speed stability experiments have been carried out on flat plates [7-9], and cones [10-12]. Each experimental facility has its own problems, some of which are due to sound radiation from the tunnel-wall boundary layer which affects the disturbance growth and dominates the transition process. Consequently, one expects to find difficulties and discrepances when comparing theoretical and experimental results for high-speed boundary layers; see Bushnell [13] for a description of the possible difficulties as well as suggested remedies. Almost all 
experimental measurements reported on the stability of boundary layers were made by means of hot-wire anemometers. Any such measurements encounter two basic difficulties that come from the response and the sensitivity of the hot wire. The hot-wire response is a combination of mass-flow and stagnation temperature fluctuations. When the hot wire is operated at high constant overheat, its response is proportional to the mean square of the mass-flow fluctuations. Also the sensitivity of the hot wire changes when it moves across the boundary layer, or whenever the mean flow is changed. Disturbances that develop in a growing boundary layer have eigenfunctions of nonsimilar nature, thus making it difficult to separate the actual growth of the disturbance from the change in the amplitude distribution across the boundary layer. Hence, each measurement technique will necessarily include the contribution of the streamwise distortion of the eigenfunction in the measurement of the growth rate of the disturbance.

Nonparallel stability theories for a compressible boundary layer were developed by ElHady and Nayfeh [14], El-Hady [15], and Gaponov [16]. The nonparallel theory accounts for the weak dependence of the flow parameters on the streamwise coordinate, as well as the velocity normal to the wall. This theory gives a more accurate mathematical model for the development of the disturbance. The calculation of the neutral stability in the nonparallel theory encounters the difficulty of being dependent upon the disturbance flow quantity e.g. streamwise velocity, mass flow, total temperature, kinetic energy, and also upon the coordinate normal to the wall. Some of these quantities can easily be measured in an experiment, while some are difficult to measure. For meaningful comparison with the experiment, the same disturbance quantity used in the measurements must be used in the stability calculations.

The purpose of this paper is to revisit the nonparallel stability theory and clarify a rather confused numerical situation. This situation arises due to totally neglecting the distortion of the eigenfunction of the disturbance when comparing with low speed experiments $[17,18]$, or due to insufficient grid resolution in calculating the distortion of the disturbance eigensolution as Mach number increases [14], which was corrected by El-Hady [15] for Mach number 4.5. The effect of the growth of the boundary layer on the amplification rates of the disturbances, and 
on the maximum amplified frequencies is assessed for a large range of Mach numbers including first- and second-mode disturbances. Disturbance growth rates are calculated based on three different methods, namely, following the maximum of the mass-flow disturbance, using the integral of the disturbance kinetic energy, and using the integral of the square of the amplitude of the mass-flow disturbance. In Section 2, the multiple scaling analysis of the stability problem is developed. Section 3 discusses different definitions of the disturbance growth rate. Results of subsonic and supersonic stability are discussed in Section 4.1. Results of hypersonic stability are discussed in section 4.2. Section 4.3 gives a comparison between nonparallel stability results and experiments. We end with concluding remarks.

\section{ANALYSIS}

In this study we are concerned with the so-called first-mode and second-mode instability of the supersonic and hypersonic flat plate boundary layers. The most amplified wave is three-dimensional (3D) for supersonic boundary layers, and two-dimensional (2D) for hypersonic boundary layers.

The flow field is governed by the laminar, compressible, 3D Navier-Stokes and energy equations. Lengths, velocities, and time are made dimensionless using a reference length $L^{*}=\left(v_{0 e}^{*} x^{*} / u_{0 e}^{*}\right)^{1 / 2}$, the local free stream velocity $u_{0 e}^{*}$, and $L^{*} / u_{0 e}^{*}$ respectively, so that the Reynolds number is given by $R=u_{0 e}^{*} L^{*} / v_{0 e}^{*}=\sqrt{R_{x}}$. Here $x^{*}$ is the streamwise distance from the leading edge, $v_{0 e}^{*}$ is the kinematic viscosity coefficient calculated at the local free stream conditions, and the asterisk indicates a dimensional quantity. The pressure is made dimensionless using $\rho_{0 e}^{*} u_{0 e}^{*}$. The temperature, density, specific heats, viscosity, and thermal conductivity of air (treated as perfect gas) are made dimensionless using their corresponding local free stream values.

We consider the 2D compressible mean flow to be slightly nonparallel; that is, the normal velocity component $v_{0}$ is small compared with the streamwise component $u_{0}$, the mean-flow quantities are weakly varying functions of the streamwise coordinate $x$. The parameter $\varepsilon$ 
characterizes this weak variation and is identified with $1 / R$. The method of multiple scales [19] is used to introduce the slow scales $x_{1}=\varepsilon x$ and $z_{1}=\varepsilon z$ in the streamwise and spanwise directions, respectively. These scales govern the growth of the boundary layer, the modulation of the disturbance amplitude, and the change in the eigenfunction, while the phase of the disturbance changes over the spatial scales $x, z$, and the temporal scale $t$. The mean-flow velocity and pressure fields are then given by

$$
u_{0}=u_{0}\left(x_{1}, y\right), \quad v_{0}=\varepsilon v_{0}\left(x_{1}, y\right), \quad p_{0}=p_{0}\left(x_{1}\right)
$$

and the mean-flow temperature, viscosity, and density are given by

$$
T_{0}=T_{0}\left(x_{1}, y\right), \quad \mu_{0}=\mu_{0}\left(x_{1}, y\right), \quad \rho_{0}=\rho_{0}\left(x_{1}, y\right)
$$

The boundary-layer temperature and density profiles are related by $\rho_{0} T_{0}=1$, and the viscosity $\mu_{0}$ is a function of the temperature only, through Sutherland's law.

To study the stability of the compressible nonparallel mean flow, we assume that the flow quantities possess a uniformly valid expansions of the form

$$
\hat{q}=q_{0}\left(x_{1}, y\right)+\sum_{m=1}^{2} \varepsilon^{m} q_{m}\left(x, x_{1}, y, z, z_{1}, t, t_{1}\right)+O\left(\varepsilon^{3}\right)
$$

where $q_{0}$ stands for the steady mean-flow quantities $u_{0}, v_{0}, w_{0}, p_{0}, T_{0}, \mu_{0}$, and $\rho_{0}\left(w_{0}=0\right.$ for 2D mean flows), $q_{1}$ and $q_{2}$ stand for the corresponding unsteady small disturbance quantities and $t_{1}=\varepsilon t$ is a slow time scale.

Substituting Eq.(1) into the governing Navier-Stokes equations, transforming the space and time derivatives to $x_{1}, z_{1}$, and $t_{1}$, subtracting the mean-flow terms, linearizing the equations, and equating the coefficients of like powers of $\varepsilon$, we obtain the following first- and secondorder disturbance equations:

Order $\varepsilon$ :

$$
L_{1}\left(u_{1}, v_{1}, w_{1}, \rho_{1}\right)=\frac{\partial \rho_{1}}{\partial t}+u_{0} \frac{\partial \rho_{1}}{\partial x}+\frac{\partial \rho_{0}}{\partial y} v_{1}+\rho_{0}\left(\frac{\partial u_{1}}{\partial x}+\frac{\partial v_{1}}{\partial y}+\frac{\partial w_{1}}{\partial z}\right)=0
$$




$$
\begin{aligned}
& L_{2}\left(u_{1}, v_{1}, w_{1}, p_{1}, T_{1}\right)=\rho_{0}\left(\frac{\partial u_{1}}{\partial t}+u_{0} \frac{\partial u_{1}}{\partial x}+\frac{\partial u_{0}}{\partial y} \nu_{1}\right)+\frac{\partial p_{1}}{\partial x}-\frac{1}{R}\left[\mu_{0} \nabla^{2} u_{1}+\frac{\partial \mu_{0}}{\partial y}\left(\frac{\partial u_{1}}{\partial y}+\frac{\partial v_{1}}{\partial x}\right)\right. \\
& \left.+\frac{\partial}{\partial y}\left(\frac{\partial u_{0}}{\partial y} \mu_{1}\right)+(m+1) \mu_{0} \frac{\partial}{\partial x}\left(\frac{\partial v_{1}}{\partial y}+\frac{\partial w_{1}}{\partial z}\right)\right]=0 \\
& L_{3}\left(u_{1}, v_{1}, w_{1}, p_{1}, T_{1}\right)=\rho_{0}\left(\frac{\partial v_{1}}{\partial t}+u_{0} \frac{\partial v_{1}}{\partial x}\right)+\frac{\partial p_{1}}{\partial y}-\frac{1}{R}\left[\mu_{0} \nabla^{2} v_{1}+\frac{\partial \mu_{0}}{\partial y}\left[m \frac{\partial u_{1}}{\partial x}+(m+2) \frac{\partial \nu_{1}}{\partial y}+m \frac{\partial w_{1}}{\partial z}\right]\right. \\
& \left.+(m+1) \mu_{0} \frac{\partial}{\partial y}\left(\frac{\partial u_{1}}{\partial x}+\frac{\partial w_{1}}{\partial z}\right)+\frac{\partial u_{0}}{\partial y} \frac{\partial \mu_{1}}{\partial x}\right)=0 \\
& L_{4}\left(u_{1}, v_{1}, w_{1}, p_{1}\right)=\rho_{0}\left(\frac{\partial w_{1}}{\partial t}+u_{0} \frac{\partial w_{1}}{\partial x}\right)+\frac{\partial p_{1}}{\partial z}-\frac{1}{R}\left(\mu_{0} \nabla^{2} w_{1}+\frac{\partial \mu_{0}}{\partial y}\left(\frac{\partial w_{1}}{\partial y}+\frac{\partial v_{1}}{\partial z}\right)\right. \\
& \left.+(m+1) \mu_{0} \frac{\partial}{\partial z}\left(\frac{\partial u_{1}}{\partial x}+\frac{\partial v_{1}}{\partial y}\right)\right]=0 \\
& L_{5}\left(u_{1}, v_{1}, p_{1}, T_{1}\right)=\rho_{0}\left(\frac{\partial T_{1}}{\partial t}+u_{0} \frac{\partial T_{1}}{\partial x}+\frac{\partial T_{0}}{\partial y} \nu_{1}\right)-(\gamma-1) M_{\infty}^{2}\left(\frac{\partial p_{1}}{\partial t}+u_{0} \frac{\partial p_{1}}{\partial x}+\frac{1}{R}\left[2 \mu_{0} \frac{\partial u_{0}}{\partial y}\left(\frac{\partial u_{1}}{\partial y}+\frac{\partial v_{1}}{\partial x}\right)\right.\right. \\
& \left.\left.+\left(\frac{\partial u_{0}}{\partial y}\right)^{2} \mu_{1}\right]\right)-\frac{1}{R \Gamma}\left[\mu_{0} \nabla^{2} T_{1}+\frac{\partial \mu_{0}}{\partial y} \frac{\partial T_{1}}{\partial y}+\frac{\partial}{\partial y}\left(\frac{\partial T_{0}}{\partial y} \mu_{1}\right)\right]=0 \\
& L_{6}\left(p_{1}, T_{1}, \rho_{1}\right)=\gamma M_{\Delta}^{2} p_{1}-\rho_{0} T_{1}-T_{0} \rho_{1}=0
\end{aligned}
$$

Order $\varepsilon^{2}$ :

$$
\begin{gathered}
L_{1}\left(u_{2}, v_{2}, w_{2}, T_{2}\right)=-\frac{\partial \rho_{1}}{\partial t_{1}}-\rho_{0}\left(\frac{\partial u_{1}}{\partial x_{1}}+\frac{\partial w_{1}}{\partial z_{1}}\right)-u_{0} \frac{\partial \rho_{1}}{\partial x_{1}}-\left(\frac{\partial u_{0}}{\partial x_{1}}+\frac{\partial v_{0}}{\partial y}\right) \rho_{1}-v_{0} \frac{\partial \rho_{1}}{\partial y}-\frac{\partial \rho_{0}}{\partial x_{1}} u_{1} \\
L_{2}\left(u_{2}, v_{2}, w_{2}, p_{2}, T_{2}\right)=-\rho_{0}\left(\frac{\partial u_{1}}{\partial t_{1}}+u_{0} \frac{\partial u_{1}}{\partial x_{1}}\right)-\frac{\partial p_{1}}{\partial x_{1}}+\frac{1}{R}\left[\mu _ { 0 } \left[(m+2)\left(\frac{\partial^{2} u_{1}}{\partial x \partial x_{1}}+\frac{\partial^{2} u_{1}}{\partial x_{1} \partial x}\right)+\frac{\partial^{2} u_{1}}{\partial z \partial z_{1}}\right.\right.
\end{gathered}
$$




$$
\begin{gathered}
\left.+\frac{\partial^{2} u_{1}}{\partial z_{1} \partial z}+m\left(\frac{\partial^{2} w_{1}}{\partial x_{1} \partial z}+\frac{\partial^{2} w_{1}}{\partial x \partial z_{1}}\right)+\frac{\partial^{2} w_{1}}{\partial z \partial x_{1}}+\frac{\partial^{2} w_{1}}{\partial z_{1} \partial x}+(m+1) \frac{\partial^{2} v_{1}}{\partial x_{1} \partial y}\right]+\frac{\partial \mu_{0}}{\partial y} \frac{\partial v_{1}}{\partial x_{1}} \\
\left.+\frac{\partial \mu_{0}}{\partial x_{1}}\left[(m+2) \frac{\partial u_{1}}{\partial x}+m \frac{\partial v_{1}}{\partial y}+m \frac{\partial w_{1}}{\partial z}\right]+\left[(m+2) \frac{\partial u_{0}}{\partial x_{1}}+m \frac{\partial v_{0}}{\partial y}\right] \frac{\partial \mu_{1}}{\partial x}\right] \\
-\left(u_{0} \frac{\partial u_{0}}{\partial x_{1}}+v_{0} \frac{\partial u_{0}}{\partial y}\right) \rho_{1}-\rho_{0}\left(\frac{\partial u_{0}}{\partial x_{1}} u_{1}+v_{0} \frac{\partial u_{1}}{\partial y}\right)
\end{gathered}
$$

$$
\begin{gathered}
L_{3}\left(u_{2}, v_{2}, w_{2}, p_{2}, T_{2}\right)=-\rho_{0}\left(\frac{\partial v_{1}}{\partial t_{1}}+u_{0} \frac{\partial v_{1}}{\partial x_{1}}\right)+\frac{1}{R}\left[\mu_{0}\left(\frac{\partial^{2} v_{1}}{\partial x \partial x_{1}}+\frac{\partial^{2} v_{1}}{\partial x_{1} \partial x}+\frac{\partial^{2} v_{1}}{\partial z \partial z_{1}}+\frac{\partial^{2} v_{1}}{\partial z_{1} \partial z}\right)+m \frac{\partial \mu_{0}}{\partial y}\left(\frac{\partial u_{1}}{\partial x_{1}}+\frac{\partial w_{1}}{\partial z_{1}}\right)\right. \\
+(m+1) \mu_{0} \frac{\partial}{\partial y}\left(\frac{\partial u_{1}}{\partial x_{1}}+\frac{\partial w_{1}}{\partial z_{1}}\right)+\frac{\partial u_{0}}{\partial y} \frac{\partial \mu_{1}}{\partial x_{1}}+\frac{\partial \mu_{0}}{\partial x_{1}}\left(\frac{\partial u_{1}}{\partial y}+\frac{\partial v_{1}}{\partial x}\right)+\left[(m+2) \frac{\partial v_{0}}{\partial y}+m \frac{\partial u_{0}}{\partial x_{1}}\right] \frac{\partial \mu_{1}}{\partial y} \\
\left.+\left[(m+1) \frac{\partial^{2} u_{0}}{\partial y \partial x_{1}}+(m+2) \frac{\partial^{2} v_{0}}{\partial y^{2}}\right] \mu_{1}\right]-\rho_{0} \frac{\partial}{\partial y}\left(v_{0} v_{1}\right)
\end{gathered}
$$

$$
\begin{aligned}
L_{4}\left(u_{2}, v_{2}, w_{2}, p_{2}, T_{2}\right)= & -\rho_{0}\left(\frac{\partial w_{1}}{\partial t_{1}}+u_{0} \frac{\partial w_{1}}{\partial x_{1}}\right)-\frac{\partial p_{1}}{\partial z_{1}}+\frac{1}{R}\left[\mu _ { 0 } \left[\frac{\partial^{2} w_{1}}{\partial x \partial x_{1}}+\frac{\partial^{2} w_{1}}{\partial x_{1} \partial x}+(m+2)\left(\frac{\partial^{2} w_{1}}{\partial z \partial z_{1}}+\frac{\partial^{2} w_{1}}{\partial z_{1} \partial z}\right)\right.\right. \\
& \left.+m\left(\frac{\partial^{2} u_{1}}{\partial z \partial x_{1}}+\frac{\partial^{2} u_{1}}{\partial z_{1} \partial x}\right)+\frac{\partial^{2} u_{1}}{\partial x_{1} \partial z}+\frac{\partial^{2} u_{1}}{\partial x \partial z_{1}}+(m+1) \frac{\partial^{2} v_{1}}{\partial z_{1} \partial y}\right] \\
+ & \left.\frac{\partial \mu_{0}}{\partial y} \frac{\partial v_{1}}{\partial z_{1}}+\frac{\partial \mu_{0}}{\partial x_{1}}\left(\frac{\partial w_{1}}{\partial x}+\frac{\partial u_{1}}{\partial z}\right)+m\left(\frac{\partial u_{0}}{\partial x_{1}}+\frac{\partial v_{0}}{\partial y}\right) \frac{\partial \mu_{1}}{\partial z}\right]-\rho_{0} v_{0} \frac{\partial w_{1}}{\partial y}
\end{aligned}
$$

$$
\begin{gathered}
L_{5}\left(u_{2}, v_{2,}, p_{2}, T_{2}\right)=-\rho_{0}\left(\frac{\partial T_{1}}{\partial t_{1}}+u_{0} \frac{\partial T_{1}}{\partial x_{1}}\right)-\rho_{0}\left(\frac{\partial T_{0}}{\partial x_{1}} u_{1}+v_{0} \frac{\partial T_{1}}{\partial y}\right)+\left(u_{0} \frac{\partial T_{0}}{\partial x_{1}}+v_{0} \frac{\partial T_{0}}{\partial y}\right) \rho_{1} \\
+(\gamma-1) M_{\infty}^{2}\left(\frac{\partial p_{1}}{\partial t_{1}}+u_{0} \frac{\partial p_{1}}{\partial x_{1}}+\frac{\partial p_{0}}{\partial x_{1}} u_{1}+v_{0} \frac{\partial p_{1}}{\partial y}+2 \mu_{0} \frac{1}{R}\left[\frac{\partial u_{0}}{\partial y} \frac{\partial v_{1}}{\partial x_{1}}+(m+2)\left(\frac{\partial u_{0}}{\partial x_{1}} \frac{\partial u_{1}}{\partial x}+\frac{\partial v_{0}}{\partial y} \frac{\partial v_{1}}{\partial y}\right)\right.\right.
\end{gathered}
$$

$\left.\left.-m \frac{\partial u_{0}}{\partial x_{1}}\left(\frac{\partial v_{1}}{\partial y}+\frac{\partial w_{1}}{\partial z}\right)+m \frac{\partial v_{0}}{\partial y}\left(\frac{\partial u_{1}}{\partial x}+\frac{\partial w_{1}}{\partial z}\right)\right]\right)+\frac{1}{R \Gamma}\left[\mu_{0}\left(\frac{\partial^{2} T_{1}}{\partial x \partial x_{1}}+\frac{\partial^{2} T_{1}}{\partial x_{1} \partial x}+\frac{\partial^{2} T_{1}}{\partial z \partial z_{1}}+\frac{\partial^{2} T_{1}}{\partial z_{1} \partial z}\right)+\frac{\partial \mu_{0}}{\partial x_{1}} \frac{\partial T_{1}}{\partial x}+\frac{\partial T_{0}}{\partial x_{1}} \frac{\partial \mu_{1}}{\partial x}\right)$ 
where $\Gamma$ is Prandtl number that is a function of the mean-flow temperature, $M_{\infty}$ is the freestream Mach number, and $m=2(e-1) / 3$ is the ratio of the second to the first viscosity coefficients. $e=0$ corresponds to the Stokes hypothesis; it is taken to be 0.8 in this analysis. Moreover,

$$
\nabla^{2}=(m+2)^{a} \frac{\partial^{2}}{\partial x^{2}}+(m+2)^{b} \frac{\partial^{2}}{\partial y^{2}}+(m+2)^{c} \frac{\partial^{2}}{\partial z^{2}}
$$

The exponents $\mathrm{a}, \mathrm{b}$, and $\mathrm{c}$ take the values 1,0,0 for $\mathrm{Eq}(3), 0,1,0$ for $\mathrm{Eq}$ (4), 0,0,1 for Eq (5), and $0,0,0$ for $\mathrm{Eq}(6)$. The leading-order equations are homogeneous and govern the disturbance motion in a parallel flow, while the higher-order equations include the nonparallel effects as inhomogeneous terms.

\section{A. First-order equations}

We express the solution of the homogeneous equations as

$$
q_{1 n}=A\left(x_{1}, z_{1}, t_{1}\right) \zeta_{n}\left(x_{1}, y, z_{1}\right) e^{i \tau}+c c . \quad n=1, \ldots, 8
$$

where $A\left(x_{1}, z_{1}, t_{1}\right)$ is an amplitude modulation function that is determined by the solvability condition of the higher-order inhomogeneous equations, $\zeta_{n}\left(x_{1} y, z_{1}\right)$ are eigenfunctions, $q_{1 n}$ are the physical wave amplitudes, and "cc" stands for complex conjugate. Note that the quantity $A\left(x_{1}, z_{1}, l_{1}\right) \zeta_{n}\left(x_{1} y_{, 2}\right)$ is unique and is independent of the eigenfunction normalization, and can be directly compared with the experiment. In Eq (13), $q_{1 n}, n=1, \ldots, 8$ stands for $u_{1}, \partial u_{1} / \partial y, v_{1}, p_{1}$, $T_{1}, \partial T_{1} / \partial y, w_{1}$, and $\partial w_{1} / \partial y$, respectively, and

$$
\frac{\partial \tau}{\partial x}=\alpha\left(x_{1}, z_{1}\right), \quad \frac{\partial \tau}{\partial z}=\beta\left(x_{1}, z_{1}\right), \quad \frac{\partial \tau}{\partial t}=-\omega
$$

The phase function $\tau$ is assumed to be continuously differentiable; that is, $\partial \alpha / \partial z_{1}=\partial \beta / \partial x_{1}$. $\alpha$ and $\beta$ are the complex wavenumbers in the $x$ and $z$ directions given by $\alpha=\alpha_{r}+i \alpha_{i}$ and $\beta=\beta_{r}+i \beta_{i}$, and $\omega$ is the complex frequency given by $\omega=\omega_{r}+i \omega_{i}$. The density disturbance is related to the pressure and temperature disturbances through the state equation (7), while the viscosity disturbance is related to the temperature disturbance by $\mu_{1}=\left(d \mu_{0} / d T_{0}\right) T_{1}$. 
Substituting (13) and (14) into the first-order equations, and writing the result as a system of eight first-order ordinary differential equations, we obtain

$$
D \zeta_{n}-\sum_{j=1}^{8} b_{n j} \zeta_{j}=0, \quad n=1, \ldots, 8
$$

subject to the boundary conditions

$$
\begin{gathered}
\zeta_{1}=\zeta_{3}=\zeta_{5}=\zeta_{7}=0, \text { at } y=0 \\
\zeta_{1}, \zeta_{3}, \zeta_{5}, \zeta_{7} \rightarrow 0, \text { as } y \rightarrow \infty,
\end{gathered}
$$

where $D=d / d y$ and the nonzero elements of $b_{n j}$ are given in Appendix A.

\section{B. Second-order Equations}

In order to determine the amplitude $A$ in (13), we seek a particular solution for the second-order equations in the form

$$
q_{2 n}=\Psi_{n}\left(y ; x_{1}, z_{1}, l_{1}\right) e^{i \tau}+c c . \quad n=1, . ., 8
$$

where $q_{2 n}$ stands for $u_{2}, \partial u_{2} / \partial y, v_{2}, p_{2}, T_{2}, \partial T_{2} / \partial y, w_{2}$, and $\partial w_{2} / \partial y$, respectively. By using Eqs (13), (14) and (18), we can write the second-order problem as eight first-order equations in the form

$$
D \psi_{n}-\sum_{j=1}^{8} b_{n j} \psi_{j}=I_{n,}, \quad n=1, \ldots, 8
$$

subject to the boundary conditions

$$
\begin{gathered}
\psi_{1}=\psi_{3}=\psi_{5}=\psi_{7}=0, \text { at } y=0, \\
\psi_{1}, \psi_{3}, \psi_{5}, \psi_{7} \rightarrow 0, \text { as } y \rightarrow \infty
\end{gathered}
$$

The coefficients $b_{n j}$ are the same as in Eq.(15), while the inhomogeneous terms $I_{n}$ are functions of the first-order eigensolution $\zeta_{n}$ of Eq.(4), $\alpha, \beta, \omega$, and the mean-flow quantities. The terms $I_{n}$ include all leading-order nonparallel flow effects. 
The necessary and sufficient condition for solving the inhomogeneous set of Eqs.(19) is that $I_{n}$ must be orthogonal to the solution of the adjoint problem. This can be expressed as

$$
\int_{0}^{\infty} \sum_{n=1}^{8} I_{n} \zeta_{n}^{*} d y=0
$$

where $\zeta_{n}^{*}$ are eigensolutions of the equation adjoint to (19). Thus $\zeta_{n}^{*}$ satisfy

$$
\begin{aligned}
& D \zeta_{n}^{*}-\sum_{j=1}^{8} \vec{b}_{n j} \zeta_{j}^{*}=0, \quad n=1, \ldots, 8, \\
& \zeta_{2}^{*}=\zeta_{4}^{*}=\zeta_{6}^{*}=\zeta_{8}^{*}=0, \text { at } y=0, \\
& \zeta_{2}^{*}, \zeta_{4}^{*}, \zeta_{6}^{*}, \zeta_{8}^{*} \rightarrow 0, \text { as } y \rightarrow \infty
\end{aligned}
$$

and $\bar{b}_{n j}=-b_{j n}$. The solvability condition (22) gives the following differential equation for the evolution of the amplitude $A$ in time and space:

$$
\frac{\partial A}{\partial t_{1}}+h_{1} \frac{\partial A}{\partial x_{1}}+h_{2} \frac{\partial A}{\partial z_{1}}+h_{3} A=0
$$

Here, $h_{1}$ and $h_{2}$ are the components of the disturbance group velocity in the $x$ and $z$ directions, respectively, while $h_{3}$ reflects the effect of the nonparallelism of the mean flow. In Eq (26), $h_{3}$ contains the $x_{1}$ and $z_{1}$ derivatives of the steady mean-flow quantities, the derivatives of the wavenumbers $\alpha$ and $\beta$, and the derivatives of the eigenfunctions $\zeta_{n}, n=1, . .8$ of the leading-order problem. The expressions for $h_{1}$ and $h_{3}$ are given in Appendix B.

For the purpose of our investigation, and to be closer in representing the experiment, we consider the spatial modulation of a single frequency disturbance (i.e. $\partial A / \partial t_{1}=0$ ) which has a uniform amplitude in the spanwise coordinate (i.e. $\partial A / \partial z_{1}=0$ ). Note that the phase of the disturbance varies with the streamwise and the spanwise directions but the slow modulation of the disturbance occurs only in the streamwise direction. Equation (26) simplifies to

$$
\frac{d A}{d x_{1}}=i \propto A, \quad \alpha=i \frac{h_{3}}{h_{1}}
$$

and therefore, 


$$
A=A_{0} \exp \left[i \int_{x_{0}}^{x} \varepsilon \alpha d x\right]
$$

where $A_{0}$ is the initial amplitude at $x=x_{0}$. In order to calculate $\alpha$, it is necessary to evaluate the nonparallel terms $\partial \zeta_{n} / \partial x_{1}$ and $d \alpha / d x_{1}$ in $h_{3}$. We differentiate the first-order equations (15) with respect to $x_{1}$ and obtain

$$
\begin{gathered}
D\left[\frac{\partial \zeta_{n}}{\partial x_{1}}\right]-\sum_{j=1}^{8} b_{n j} \frac{\partial \zeta_{j}}{\partial x_{1}}=\sum_{j=1}^{8} \frac{\partial b_{n j}}{\partial x_{1}} \zeta_{j}, \quad n=1, \ldots, 8 \\
\frac{\partial \zeta_{1}}{\partial x_{1}}=\frac{\partial \zeta_{3}}{\partial x_{1}}=\frac{\partial \zeta_{5}}{\partial x_{1}}=\frac{\partial \zeta_{7}}{\partial x_{1}}=0, \text { at } y=0, \\
\frac{\partial \zeta_{1}}{\partial x_{1}}, \frac{\partial \zeta_{3}}{\partial x_{1}}, \frac{\partial \zeta_{5}}{\partial x_{1}}, \frac{\partial \zeta_{7}}{\partial x_{1}} \rightarrow 0, \text { as } y \rightarrow \infty
\end{gathered}
$$

The homogeneous part of Eq.(29) has a nontrivial solution; its eigenvalues and adjoint is the same as that for the first-order equations (15). Again, by applying the solvability condition, we can evaluate $d \alpha / d x_{1}$, and then integrate Eqs (29)-(31) to determine $\partial \zeta_{n} / \partial x_{1}$, using the same procedure as for the first-order equations, but for a nonhomogeneous set of equations.

Therefore, to the leading-order approximation Eqs (13), (14), and (28) give the disturbance as

$$
q_{n}=A_{0} \zeta_{n}\left(x_{1}, y\right) \exp i\left[\int(\alpha+\varepsilon \alpha) d x+\beta z-\omega t\right]+O\left(\varepsilon^{2}\right), \quad n=1, \ldots, 8
$$

Where both $\beta$ and $\omega$ in Eq (32) are real constants.

The spatial growth rate of the disturbance can be given as:

$$
\sigma_{n}=-\alpha_{i}-\varepsilon \alpha_{i}+\varepsilon\left(\frac{1}{\zeta_{n}} \frac{\partial \zeta_{n}}{\partial x_{1}}\right)_{r}, \quad n=1, \ldots, 8
$$

The first term is the spatial growth rate in a parallel flow, while the second and third terms are the nonparallel correction. A correction to the disturbance wavenumber due to mean-flow nonparallelism can also be calculated by the theory. The wavenumber of the disturbance is given by : 


$$
k_{n}=\alpha_{r}+\varepsilon \alpha_{r}+\varepsilon\left[\frac{1}{\zeta_{n}} \frac{\partial \zeta_{n}}{\partial x_{1}}\right]_{i}, \quad n=1, \ldots, 8
$$

where the subscripts $r$ and $i$ in Eqs (32) and (33) stand for the real and imaginary parts of the quantity.

\section{DEFINITION OF THE GROWTH RATE}

Equation (13) gives the physical disturbance amplitude as $A\left(x_{1}\right) \zeta\left(x_{1}, y\right)$, where $A\left(x_{1}\right)$ is the amplitude modulation function, and $\zeta\left(x_{1}, y\right)$ is an eigenfunction. Because part of $\zeta$ can be absorbed in $A$, and hence in $\alpha$ in $\mathrm{Eq}(27)$, then $\alpha$ is dependent on the normalization of the eigenfunction $\zeta$. On the other hand, the physical disturbance amplitude $A\left(x_{1}\right) \zeta\left(x_{1}, y\right)$ is unique and is independent of the eigenfunction normalization. The spatial growth rate of the disturbance then can be evaluated by using $\mathrm{Eq} \mathrm{(33)} \mathrm{which} \mathrm{consists} \mathrm{of} \mathrm{three} \mathrm{terms.} \mathrm{The} \mathrm{first} \mathrm{term} \mathrm{is}$ the parallel growth rate, while the second and the third terms together give the nonparallel correction to the parallel growth rate. The second term is independent of $y$, while the third term, which arises due to the distortion of the eigenfunction, is not only $x$ and $y$ dependent, but

also is a function of the physical-flow quantity. Because of this behavior, the neutral stability curve as well as the growth rate of the disturbance in the nonparallel stability theory becomes a function of $(x, y)$ as well as the flow quantity used in the calculations. This complicated character of the growth rate and of the stability definition, although undesirable, is shared by the experiments.

All growth rate calculations reported in this paper use the definition given by Eq (33), which is based on relevant physical quantities. The mass-flow disturbance is chosen for this purpose as a physical quantity that can be measured experimentally in compressible boundary layers. The mass-flow disturbance $\zeta_{9}\left(x_{1}, y\right)$ is derived by subtracting the mean mass flow from the total mass flow and linearizing to arrive at

$$
\zeta_{9}\left(x_{1}, y\right)=\frac{u_{0}}{T_{0}}\left[\gamma M_{\infty}^{2} \zeta_{4}-\frac{\zeta_{5}}{T_{0}}\right]+\frac{\zeta_{1}}{T_{0}}
$$


where $\zeta_{1}, \zeta_{4}$ and $\zeta_{5}$ are $u_{1}, p_{1}$, and $T_{1}$ respectively. We normalize the eigensolutions such that the maximum of $\left|\zeta_{9}\right|$ over the normal coordinate is equal to one.

At Mach numbers 1.6 and 3.0, and different streamwise locations, Figs (1) and (2) show the variation with the normal coordinate of the mass-flow disturbance amplitude across the boundary layer, the variation of the mass-flow eigenfunction distortion (the third term in Eq (33)) and the variation of the total nonparallel growth rate given by Eq (33). These figures show that the distortion of the mass-flow eigenfunction can have an apparent stabilizing or destabilizing influence depending on the $y$-location where the growth rate is calculated. The value of the distortion is relatively small at the $y$-location where $\left|\zeta_{9}\right|$ is maximum.

The rapid change of the distortion term that is observed in Figs (1) and (2) around the peak of the eigenfunction makes the theoretical calculations of nonparallel growth rates highly sensitive to both the grid resolution in the normal coordinate, as well as the procedure used to search for the peak value. This sensitivity increases as Mach number increases. The nonparallel results of El-Hady and Nayfeh [14] suffer from this sensitivity due to the use of low grid resolution in the normal direction. Note that the eigenvalue of the first-order problem (15) is not sensitive to the grid resolution. These results were corrected by El-Hady [15] for Mach number 4.5. The rapid change in the distortion term may easily create a source of discrepancy between nonparallel-theory calculations and experiments that claim to follow a certain $y$ location (within the experimental error). This point will be discussed in more details in Section 4.3 when comparing the theoretical and the experimental results. To decrease the effect of this sensitivity in calculating the nonparallel growth rate, a value of the distortion effect may be chosen near the outer edge of the boundary layer, where it is apparently constant as shown in Figs (1) and (2). However, it might be difficult in the experiment to measure with reasonable accuracy the amplitude of the disturbance at these $y$-locations.

Previous nonparallel stability investigations using the same analysis approach dropped the third term in $\mathrm{Eq}$ (33) when calculating the nonparallel growth rate $[14,17,18]$. This was justified in [17] by claiming that the contribution of the distortion of the eigenfunction to the 
growth rate can be neglected when the growth rate is calculated at $\left|\zeta_{1}\right|_{\max }$. It was also claimed in $[17,18]$ that, by dropping the third term in Eq (33), the agreement between the theoretical and the experimental growth rates becomes better for the experimental data that are measured at or near $\left|\zeta_{1}\right|_{\max }$. Both justifications are in error. With respect to the first claim, it is true that the distortion of the eigenfunction is near minimum when it is calculated at $\left|\zeta_{1}\right|_{\max }\left(\right.$ or $\left|\zeta_{9}\right|_{\max }$ for compressible flows) but its value cannot be neglected compared to other terms in Eq (33). To show this fact, Table 1 lists calculated values of different terms in Eq (33) for the most amplified nonparallel frequency at $R=1000$. At $M_{\infty}=0$, Van Stijn and Van de Vooren [20] corrected the error in the growth rate calculations by adding the third term in Eq (33). With respect to the experiments and the comparison with the theory, difficulties encountered at low speeds and discussed by Saric [6] may have large effects on the experimental measurements. Consequently, these experimental data, especially for high frequency disturbances, should be treated cautiously. Sensitivity studies performed by Bertolotti [5] indicated that many factors may contribute to large deviation in the measurements and to the discrepancy between theoretical and experimental results .

The variation of the disturbance growth rate with the normal coordinate as defined by (33) suggests the use of a quantity that is integrated across the boundary layer. The square of the mass-flow amplitude, or the total energy of the disturbance may be suitable physical quantities that can be used for that purpose. While the first is measurable, the second is difficult to measure due to lack of adequate means for measuring the disturbance normal velocity component [6]. In this paper, we also present results of the disturbance growth rates using the integral of these physical quantities in Eq (33) to calculate the nonparallel growth rates. The integral of the square of the mass-flow disturbance is given by,

$$
\hat{E}(x)=\int_{0}^{\infty}\left[\zeta_{9} \bar{\zeta}_{9}\right] d y
$$

and the integral of the disturbance kinetic energy is defined as ,

$$
E(x)=\int_{0}^{\infty}\left[\frac{2 u_{0}}{T_{0}} \zeta_{1}+\frac{1}{T_{0}}\left(\zeta_{1} \bar{\zeta}_{1}+\zeta_{3} \bar{\zeta}_{3}+\zeta_{7} \bar{\zeta}_{7}\right)+\frac{u_{0}^{2}}{T_{0}}\left(\gamma M_{\infty}^{2} \zeta_{4}-\frac{\zeta_{5}}{T_{0}}\right)\right] d y
$$




\section{RESULTS AND DISCUSSIONS}

\subsection{Subsonic and supersonic stability}

The effect of mean-flow nonparallelism on the boundary- layer stability is demonstrated in this section by presenting results for the disturbance growth rates at two streamwise locations, namely, $R=500$, and $R=1000$. Results are presented in Figs. (3a) and (3b) for Mach numbers 0.0 and 0.8 where a 2D Tollmien Schlishting (TS) mode is dominant, and in Figs (4a)-(4c) for Mach numbers 1.6, 2.2, and 3.0, where an oblique first mode is dominant at an angle $\theta$ between 50-65 degrees. Each figure shows three different curves for the growth rate. Curve (a) is the parallel spatial growth rate ( first term in Eq 33), curve (b) is the nonparallel spatial growth rate without the distortion effect of the eigenfunction (first two terms in Eq (33)), and curve (c) is the total nonparallel spatial growth rate including the contribution of the distortion of the eigenfunction. The growth rates (a) and (b) are independent of the normal coordinate $y$, as well as the disturbance-flow quantity, but the growth rate (c) is a function of both. In Figs (3) and (4), we choose to calculate the growth rate (c) at the $y$-location where the mass-flow disturbance amplitude peaks. This choice is made in order to simulate the experimental measurements at high speed boundary layers which use a hot wire operated at constant overheat to follow the peak of the mass-flow disturbance.

At subsonic Mach numbers, Fig (3), curve (b) overestimates the nonparallel effects; however, curve (c) shows that if one follows $\left|\zeta_{9}\right|_{\max }$ (in the incompressible limit the mass-flow and the streamwise-velocity disturbances have identical $y$-distribution), one observes a slight shift of the unstable frequency band to higher values, but only a small effect on the growth rates when compared with the parallel curve (a).

As Mach number increases to the supersonic range, Fig (4) indicates that curve (b) still overestimates the nonparallel effects. Curve (c) that follows $\left|\zeta_{9}\right|_{\text {max }}$ shows large effects on the growth rates accompanied by a one-side shift of the unstable frequency band to higher frequencies when compared with the parallel curve (a). At Mach numbers 1.6, 2.2, and 3.0, oblique 
disturbances are used in the calculations because they are most unstable. The contribution of the flow nonparallelism at supersonic Mach numbers to the disturbance growth rates, unstable frequency band, and the shift of the most unstable frequency, seems different from that at subsonic Mach numbers. However, by comparing Fig (4a) for an oblique wave at $M_{\infty}=1.6$ with Fig (5) for a 2D wave at the same Mach number, we conclude that this difference is due to the obliqueness of the disturbance rather than the increase in Mach number.

Previous figures show the local (at fixed $R$ ) behavior of the total nonparallel growth rate, curve (c), with respect to the parallel one, curve (a). The same behavior is also observed when a disturbance with a fixed frequency propagates downstream. For $2 \mathrm{D}$ and oblique disturbances at $M_{\infty}=1.6$, Figs (6a) and (6b) show respectively the variation of growth rate curves (a) and curve (c) with Reynolds number for a nondimensional frequency $F=\omega / R=40 \times 10^{-6}$. The growth rates of the 3D disturbances in Fig (6b) are calculated with constant spanwise wavenumber parameter $B=10^{3} \beta / R=0.1$ in the streamwise direction corresponding to a wave angle of 50 degrees at branch $\mathrm{I}$. The wave angle slightly increases as the disturbance propagates downstream. The solid dots in Fig (6) are the results by Bertolotti (unpublished data provided in a private communication) using the parabolic stability equations approach (PSE). They are in full agreement with our results at $M_{\infty}=1.6$. The PSE approach $[5,21]$ uses the fact that the second-order derivatives of the disturbances growth rate, wavelength, and velocity in the streamwise direction are negligible, resulting in an initial value problem which is solved by a marching procedure.

Figure (7) shows the effect of the wave obliqueness on the maximum nonparallel growth rate (curve c) at $M_{\infty}=1.6$ and $R=500$. The maximum parallel growth rate (curve a) is included for comparison. Here the maximum growth rate is calculated with respect to the frequency. Nonparallel effects are almost negligible for $2 \mathrm{D}$ and slightly oblique waves and increase as the wave angle increases.

Figure (8) gives the variation of the maximum spatial growth rates with respect to frequency of an oblique disturbance at 55 degrees with streamwise position. The same variation 
is shown for a 2D disturbance for comparison. Considerable nonparallel effect is indicated by curve (c) for the oblique wave (about $20 \%$ increase in the maximum spatial growth rate at $R=1000$, while it is negligible for $2 \mathrm{D}$ disturbances.

Results concerning the integral definition of the growth rates are presented in Figs (9a) and (9b) for a 2D TS mode at Mach numbers 0 and 0.8 , respectively, and in Figs (10a)-(10c) for an oblique mode at Mach numbers 1.6, 2.2, and 3.0, respectively. Each figure shows three different curves for the disturbance growth rates. Curve (a) is the parallel spatial growth rate, curve (d) is the nonparallel spatial growth rate using the integral of the square of the mass-flow disturbance, and curve (e) is the nonparallel spatial growth rate using the integral of the disturbance kinetic energy. All of these growth rates are independent of the normal coordinate. These figures show that the integral of energy criterion always overestimates the nonparallel effect compared to other criteria. As Mach number increases, Fig (10) shows little difference between curves (d) and (e).

\subsection{Hypersonic stability}

As Mach number increases, multiple eigenvalues of amplified and damped disturbance modes result as solutions to the compressible stability equations [22]. The leading unstable mode (called second mode) and higher ones are most unstable as 2D waves.

In this section, we present disturbance growth rate results at Mach numbers 4.5, 5.8, 7.0, and 10 , concentrating on the $2 \mathrm{D}$ second mode at $R=1000$. A comparison between growth rate curves (a) parallel, (b) nonparallel without the distortion of the eigenfunction, and (c) nonparallel with the distortion of the eigenfunction, are shown in Figs (11a)-(11c) for Mach numbers 4.5, 5.8, and 7.0, respectively. Again, in these figures, the growth rate curve (c) is calculated at the $y$-location where the mass-flow disturbance is maximum. Figures (11a)-(11c) show that curve (b) overestimates the nonparallel effect, while curve (c) indicates a shift of the unstable frequency band to higher values. Although the most amplified frequency is higher due to nonparallel flow effect, the maximum growth rate is almost the same as the parallel calculations. 
Results of the integral definition of the growth rate for the second mode are given in Figs (12a)-(12c) for Mach numbers 4.5, 5.8, and 7.0, respectively. Curve (e), which represent the integral of the disturbance kinetic energy, predicts almost the same nonparallel growth as curve (c) in Fig (11). Also, curve (d) of the integral of the square of the mass-flow disturbance is underestimating the growth rates compared to curve (c) of Fig (11).

A summary of nonparallel flow effects is given in Figs (13a) and (13b) for a range of Mach numbers from $0-10$ at $R=1000$. The maximum mass-flow amplitude criterion is used to calculate the nonparallel growth rates. The maximum spatial growth rate (Fig 13a) calculated using the nonparallel theory is higher by approximately $20 \%$ in the supersonic range (for an oblique first mode), and by about $8 \%-22 \%$ in the hypersonic range (for a $2 \mathrm{D}$ second mode) as compared to the parallel one. Another noticeable nonparallel effect is shown in Fig (13b). Nonparallel calculations indicate a shift of the most unstable frequency to a higher value by about $6 \%-12 \%$ in the supersonic range, and by about $6 \%-12 \%$ in the hypersonic range compared to the parallel one.

\subsection{Comparison with experiments}

The number of experiments that measure the flat plate linear stability characteristics of supersonic or hypersonic boundary layer is limited. Serious difficulties are usually present in such experiments due to sound radiation from the turbulent wall boundary layer $[7,9,10]$ that ultimately affect the disturbance growth rate measurements (noisy wind tunnels). Another limitation of the experiments is that they study the propagation of disturbances that arise naturally in the boundary layer $[7,9,10]$. These disturbances are comprised of a frequency spectrum with unclear impact of the different components on the signal of the hot-wire anemometer. Stability theory usually studies the development of individual components of the disturbance spectrum corresponding not only to a certain frequency but also propagating at a certain angle.

With these difficulties, stability experiments were undertaken mainly to qualitatively prove the findings of the stability theory concerning the first- and second-mode characteristics in 
supersonic and hypersonic speeds. Data from supersonic experiments using naturallydeveloping disturbances are usually compared with calculations for the most theoretically amplified disturbances which are oblique. The comparison performed by El-Hady and Nayfeh [14] with these experiments cannot be regarded as correct, and the uncertainty of the wave angle in these experiments make them unsuitable for comparison with theoretical nonparallel results.

The detailed experiment of Kendall [8] at $M_{\infty}=4.5$ is superior to others $[7,9,10]$ for the following reasons. The tunnel wall boundary layers were laminar, and difficulties caused by turbulence-induced sound waves were avoided. Artificial disturbances of known frequencies and wave obliqueness angles $\theta=0^{\circ}$, and $\theta=55^{\circ}$ were introduced into the flat plate boundary layer. This was achieved by glow discharge formed using electrode pairs positioned at these angles. The glow discharge locally heats the flow producing a pressure disturbance with too small dissipating power to affect the mean flow. A hot-wire anemometer that operates at high constant overheat was used for wave measurements, thus minimizing its sensitivity to total temperature disturbances. The distribution of the measured disturbance amplitude across the boundary layer in Kendall's experiment was almost similar at different streamwise locations. Because of this, the maximum amplitude points followed in the experiment had a contour of almost constant $y$-location; hence the sensitivity of the hot wire to changes in the mean flow conditions was minimum. Then, energy fluctuations measured by the hot wire have been interpreted as being nearly proportional to the mass-flow disturbance amplitude.

In Kendall's experiment, the disturbance growth rates were measured in the streamwise direction following the maximum amplitude location. The disturbances introduced at $\theta=55^{\circ}$ were found to grow nearly equal at various spanwise locations. One minor difficulty remaining unresolved in Kendall's experiment is that the thickness of the boundary layer exceeded the theoretically predicted value by about $10 \%$, although the experimental velocity profiles were similar to the calculated ones. For comparison purposes, the calculations are made at a value of Reynolds number based on the experimental displacement thickness. 
Figure (14a) shows a comparison of the growth rates of a first mode oblique disturbance with $\theta=55^{\circ}$ at $R=1550$. Figure (14b) shows a comparison of the growth rates of a second mode 2D disturbance with $\theta=0^{0}$ at $R=1550$. Nonparallel growth rates are calculated by using Eq (33) at the $y$-location where the mass-flow disturbance has a maximum. For the first mode, nonparallel calculations (curve (c)) enhances the agreement between the theory and the experiment especially near the peak growth rates. However, higher growth rates are given by scattered experimental data in the frequency range $15-22 \times 10^{-6}$. For the second mode, Fig(14b) shows excellent agreement between the nonparallel calculations and the experiment at the lower frequency region and good agreement near the second neutral point. Also, the experimental data indicate a shift in the unstable frequency band from the parallel results, as predicted by the nonparallel calculations. However, the maximum growth rate for the second mode is not matched between the calculations and the experiment.

As mentioned in Section 3, the discrepancy between the nonparallel calculations and the experiment is expected. The wide peaks predicted by the calculations [15] of the mass-flow disturbance amplitude at some frequencies make it experimentally difficult to measure the actual growth rate, especially if the growth rate changes rapidly around the peak location. In Fig (15), we show theoretical results of the variation with $y$ of both the mass-flow disturbance amplitude $\left|\zeta_{9}\right|$ and the nonparallel growth rate $(\mathrm{Eq} 33)$ based on $\zeta_{9}$. These results are given in Fig(15) for the frequencies (a) $F=15 \times 10^{-6}$ for a first mode and (b) $F=125 \times 10^{-6}$ for a second mode, and indicate a rapid change of growth rate around the peak amplitude. These two frequencies are chosen because of the apparent uncertainty in the measurement of the experimental growth rate near these frequencies, see Fig (14). These experimental growth rates are included in Figs (15) to show that the experimental measurements of the mass-flow amplitude lies within a certain percentage deviation from the peak. The $y$-variations of the mass-flow amplitude and the growth rates shown in Fig (15) are typical for the first and second unstable frequency range at $R=1550$, except that the $y$-location of $\left|\zeta_{9}\right|_{\max }$ varies slightly with frequency. The peak is not sharp in general, but it extends over a considerable normal distance [15]. 


\section{CONCLUDING REMARKS}

The nonparallel correction to the growth rates of disturbances developing in the flat plate boundary layer have been computed for a wide range of Mach numbers. Various definitions are used to calculate the nonparallel growth rates. A definition, which is based on a pointwise measure for the disturbance amplitude, is a function of the normal as well as the streamwise coordinates. On the other hand, a definition which is based on an integrated measure (across the boundary layer) for the disturbance amplitude allows one to define a unique total growth rate at any streamwise location. From the present calculations, we conclude with the following remarks :

1. If we choose to follow the peak of the mass-flow amplitude in the calculations, the growth rate is changing rapidly around this point. Theoretical calculations of the eigenfunction and its $x$-derivative require high resolution in the normal coordinate direction in order to correctly estimate the growth rates. On the other hand, calculations at different Mach numbers show that the nonparallel growth rate $(\mathrm{Eq} 33)$ at a fixed $R$ is nearly independent of $y$ towards the outer edge of the boundary layer.

2. The distortion of the mass-flow eigenfunction (the third term in Eq 33) is small at the $y$ location where the mass-flow amplitude peaks. However, its contribution to the calculated growth rate is significant.

3. Nonparallel effects calculated at $R=1000$ by using the maximum mass-flow amplitude criterion indicate that the maximum nonparallel spatial growth rate is higher by approximately $20 \%$ in the supersonic range (for an oblique first mode), and by about $8 \%-22 \%$ in the hypersonic range (for a 2D second mode) as compared to the parallel one. Also, in the range of Mach numbers from $0-10$, the most unstable frequency shifts to a higher value by about $6 \%$ $12 \%$ compared to the parallel one. 
4. A meaningful comparison between the theory and experiment requires that the same flow physical quantity be used to calculate the disturbance growth rate in both. All terms of Eq (33) are accounted for in the experimental measurement. When the mass-flow amplitude is used for this purpose, uncertainty in detecting its peak in the experiment will contribute to errors in the experimental determination of the disturbance growth rates; and hence a discrepancy with the theoretical predictions. This uncertainty most probably occurred in Kendall's experiment [8] at $M_{\infty}=4.5$ when measuring the growth rate at some frequencies. In spite of that, we find good agreement between the calculated nonparallel growth rates and Kendall's experiment [8] at $M_{\infty}=4.5$.

\section{ACKNOWLEDGMENT}

This work was supported by the Theoretical Flow Physics Branch of NASA Langley Research Center, under contract NAS1-18599.

$$
\begin{aligned}
& \text { APPENDIX A : Nonzero elements of } b_{n j}, n=1, \ldots 8, j=1, \ldots 8 \text { in Eq (15) } \\
& b_{12}=1, \quad b_{21}=i R \phi /(\mu T)+\alpha^{2}+\beta^{2}, \\
& b_{22}=-\mu_{y} / \mu, \quad b_{23}=R u_{y} /(\mu T)-i \alpha\left[\mu_{y} / \mu+(m+1) T_{y} / T\right], \\
& b_{24}=i \alpha R / \mu-(m+1) \gamma M_{\infty}^{2} \alpha \phi, \\
& b_{25}=(m+1) \alpha \phi / \mu-\left(\rho u_{y}\right)_{y} / \mu, b_{26}=-\hat{\mu} u_{y} / \mu, \\
& b_{31}=-i \alpha, \quad b_{33}=T_{y} / T, b_{34}=-i \gamma M_{\infty}^{2} \phi, \quad b_{35}=i \phi / T, \\
& b_{37}=-i \beta, \quad b_{41}=-i \alpha \chi\left[(m+2) b_{33}-2 b_{22}\right], \quad b_{42}=\chi b_{31}, \\
& b_{43}=(m+2) \chi\left[T_{y y} / T-b_{22} b_{33}\right]-\chi b_{21}, \\
& b_{44}=-i(m+2) \chi \gamma M_{\infty}^{2}\left[\left(b_{33}-b_{22}\right) \phi+\alpha u_{y}\right], \\
& b_{45}=i \chi\left[\alpha u_{y}(\mu / \mu+(m+2) / T)+(m+2) \mu_{y} \phi /(\mu T)\right],
\end{aligned}
$$


$b_{46}=i(m+2) \chi \phi / T, \quad b_{47}=\beta b_{41} / \alpha, \quad b_{48}=-i \chi \beta$,

$b_{56}=1, \quad b_{62}=-2(\gamma-1) M_{\infty}^{2} \Gamma u_{y}$,

$b_{63}=R \Gamma T_{y} /(\mu T)+i \alpha u_{y} b_{62}$,

$b_{64}=-i R(\gamma-1) M_{\infty}^{2} \Gamma \phi / \mu$,

$b_{65}=i R \Gamma \phi /(\mu T)+\alpha^{2}+\beta^{2}-(\gamma-1) M_{\infty}^{2} \Gamma \mu u_{y}{ }^{2}-\mu_{y y} / \mu$,

$b_{66}=2 b_{22}, \quad b_{83}=b_{37}\left[(m+1) b_{33}-b_{22}\right]$,

$b_{78}=1, \quad b_{84}=\beta b_{24} / \alpha, \quad b_{85}=(m+1) \beta \phi / T$,

$b_{87}=b_{21}, \quad b_{88}=b_{22}$

where $\phi=\alpha u-\omega, \chi=1 /\left[R / \mu+i(m+2) \gamma M_{\infty}^{2} \phi\right], \hat{\mu}=d \mu / d T$, and the $y$-subscript denotes $\partial / \partial y$. The subscript 0 that is adopted in the analysis for the mean-flow quantities is dropped here for simplicity.

APPENDIX B : $h_{1}$ and $h_{3}$ in Eq 27

$h_{1}=\sum_{n=0}^{8} \int_{0}^{\infty} a_{n} \zeta_{n}^{*} d y, \quad h_{3}=\sum_{n=0}^{8} \int_{0}^{\infty} d_{n} \zeta_{n}^{*} d y$

where the nonzero elements of $a_{n}, n=1, \ldots, 8$ are :

$a_{2}=-i(m+1) \alpha T Q_{1}-R Q_{2} / \mu, \quad a_{3}=T Q_{1}$,

$a_{4}=(m+2) \chi\left[\left(\mu_{y} T / \mu+2 T_{y}\right) Q_{1}+T Q_{1_{y}}\right]+R \chi Q_{3} / \mu$

$a_{6}=-R \Gamma Q_{5} / \mu, \quad a_{8}=-i(m+1) \beta T Q_{1}-R Q_{4} / \mu$

where

$Q_{1}=-\left(\zeta_{1}+\gamma M_{\infty}^{2} u \zeta_{4}-u \zeta_{S} / T\right) / T$

$Q_{2}=[2 i(m+2) \mu \alpha / R-u / T] \zeta_{1}+\left[\mu_{y} \zeta_{3}+(m+1) \mu\left(\zeta_{3 y}+i \beta \zeta_{7}\right)\right] / R-\zeta_{4}$,

$Q_{3}=\left[m \mu_{y} \zeta_{1}+(m+1) \mu \zeta_{1}+\hat{\mu} u_{y} \zeta_{5}\right] / R+\kappa \zeta_{3}$ 
$Q_{4}=i(m+1) \mu \beta \zeta_{1} / R+\kappa \zeta_{7}$,

$Q_{5}=(\gamma-1) M_{\infty}^{2}\left(2 \mu u_{y} \zeta_{3} / R+u \zeta_{4}\right)+[2 i \mu \alpha /(R \Gamma)-u / T] \zeta_{5}$,

and the nonzero elements of $d_{n}, n=1, \ldots, 8$ are :

$d_{2}=-i(m+1) \alpha T S_{1}-R S_{2} / \mu, d_{3}=T S_{1}$,

$d_{4}=(m+2) \chi\left[\left(\mu_{y} T / \mu+2 T_{y}\right) S_{1}+T S_{1}\right]+R \chi S_{3} / \mu$,

$d_{6}=-R \Gamma S_{5} / \mu, \quad d_{8}=-i(m+1) \beta T S_{1}-R S_{4} / \mu$

where

$\left.S_{1}=\left\{\left[u_{x}+v_{y}-2 \Delta T\right] \zeta_{5}+u \zeta_{5 x}+v \zeta_{6}+T_{x} \zeta_{1}\right] / T^{2}-\gamma M_{\infty}^{2}\left[u_{x}+v_{y}-\Delta T\right] \zeta_{4}+u \zeta_{4 x}+v \zeta_{4 y}\right] / T-\zeta_{1 x} / T$

$S_{2}=\left[i(m+2)(\alpha \mu)_{x} / R-u_{x} / T\right] \zeta_{1}+[2 i(m+2) \mu \alpha / R-u / T] \zeta_{1 x}-v \zeta_{2} / T+\left[(m+1) \mu \zeta_{3 x y}+m \mu_{x} \zeta_{3 y}+\mu_{y} \zeta_{3 x}\right] / R$

$-\gamma M_{\infty}^{2} \Lambda \zeta_{\mathcal{A}} / T-\zeta_{4 x}+\left\{i \alpha \hat{\alpha}\left[(m+2) u_{x}+m v_{y}\right]+N T^{2}\right\} \zeta_{5}+i \beta\left[(m+1) \mu \zeta_{7 x}+m \mu_{x} \zeta_{7}\right] / R$

$S_{3}=\left[m \mu_{y} \zeta_{1 x}+\mu_{x} \zeta_{2}+(m+1) \mu \zeta_{2 x}\right] / R+\left[i(\alpha \mu)_{x} / R-v_{y} / T\right] \zeta_{3}-\nu \zeta_{3 y} / T+\kappa \zeta_{3 x}$

$+\left[(m+1) \hat{\mu} u_{x y}+u_{y} \hat{\mu}_{x}+(m+2) \hat{\mu} v_{y y}\right] \zeta_{5} / R+\hat{\mu} u_{y} \zeta_{5 x} / R+\left[(m+2) v_{y}+m u_{x}\right]\left(\hat{\mu} \zeta_{5}\right)_{y} / R$

$S_{4}=i \beta\left[\mu_{x} \zeta_{1}+(m+1) \mu \zeta_{1 x}\right] / R-i \beta \mu m\left(u_{x}+\nu_{y}\right) \zeta_{S} / R+i(\alpha \mu)_{x} \zeta_{7} / R+\kappa \zeta_{7 x}-\nu \zeta_{8} / T$

$S_{5}=(\gamma-1) M_{\infty}^{2}\left\{p_{x} \zeta_{1}+2 i \mu\left(\alpha \zeta_{1}+\beta \zeta_{7}\left(\left[(m+2) u_{x}+m v_{y}\right] / R-T_{x} \zeta_{1} / T\right\}\right.\right.$

$+2 \mu(\gamma-1) M_{\infty}^{2}\left[u_{x} \zeta_{3 x}+\left[m u_{x}+(m+2) v_{y}\right] \zeta_{3 y}\right]-\left(u T_{x}+v T_{y}\right)\left(\gamma M_{\infty}^{2} \zeta_{4} / T-\zeta_{5} / T^{2}\right)+(\gamma-1) M_{\infty}^{2}\left(u \zeta_{4 x}+v \zeta_{4 y}\right)$

$+i\left(\mu \alpha_{x}+2 \alpha \mu_{x}\right) \zeta_{s} /(R \Gamma)+[2 i \alpha \mu /(R \Gamma)-\mu / T] \zeta_{S_{x}}-v \zeta_{6} T$

where

$\Delta=u T_{x}+v T_{y}, \Lambda=u u_{x}+v u_{y}, \quad \kappa=2 i \mu \alpha / R-u / T$ 
and the $x$-subscript denotes $\partial / \partial x$.

\section{REFERENCES}

1. Schubauer, G. B. and Skramstad, H. K., Laminar Boundary-Layer Oscillations and Transition on a Flat Plate. J. Res. Nat. Bur. Stand., 38,1947.

2. Ross J. A., Barnes F. H., Burns, J. G., and Ross, M. A. S., The flat plate boundary layer. Part 3. Comparison of theory with experiment. J. Fluid Mech. 43, 819-832, 1970.

3. Kachanov, Yu. S., Koslov, V. V., and Levchenko, V. Ya., Growth of small disturbances in a laminar boundary layer. Ucheniye Zapiski TSAGI, vol VI, No. 5, pp.137-140, 1975 (in Russian).

4. El-Hady, N. M., Evolution of Resonant Wave Triads in Three-Dimensional Boundary Layer. Phys. Fluids A, vol.1, 1989, pp. 549-563.

5. Bertolotti, F. P., Linear and Nonlinear Stability of Boundary Layers with Streamwise Varying Properties. Ph.D. Dissertation, The Ohio State University, 1990.

6. Saric, W. S., Low Speed Experiments: Requirements for Stability Measurements. In: Instability and Transition, vol. 1, 1990, ed. M. Y. Hussaini and R. G. Voigt, Springer-Verlag.

7. Laufer, J. and Vrebalovitch, T., Stability and Transition of a Supersonic Laminar Boundary Layer on an Insulated Flat Plate. J. Fluid Mech., vol. 9, 1960, pp. 257-299.

8. Kendall, J. M., Jr., Supersonic Boundary Layer Stability Experiments. Boundary Layer Transition Study Group Meeting, vol. II- Session on Boundary-Layer Stability, W. D. McCauley, ed., BSD-TR-67-213, vol. II, U.S. Air Force, Aug. 1967, pp. 10-1 - 10-8. (Available from DTIC as AD 820364 ).

9. Demetriades, A., An Experiment on the Stability of Hypersonic Boundary Layers. J. Fluid Mech., vol. 7, 1960, pp. 385-396.

10. Kendall, J. M., Wind Tunnel Experiments Relating to Supersonic and Hypersonic Boundary-Layer Transition. AIAA J., vol. 13, 1975, pp.290-299. 
11. Demetriades, A., Hypersonic Viscous Flow Over a Slender Cone: Part III: Laminar Instability and Transition. AIAA Paper 74-535, 1974.

12. Stetson, K. F., Thompson, E. R., Donaldson, J. C. and Siler, L. G., Laminar BoundaryLayer Stability Experiments on a Cone at Mach 8. Part 1: Sharp Cone. AIAA Paper 83-1761, 1983.

13. Bushnell, D., Suggested Future Directions in High Speed Transition Experimental Research. In: Stability and Transition, vol. 1, 1990, ed. M. Y. Hussaini and R. Voigt, Springer-Verlag.

14. El-Hady, N. M. and Nayfeh, A. H., Nonparallel Stability of Compressible Boundary-Layer Flows. AIAA Paper No. 80-0277, 1980.

15. El-Hady, N. M., On the Effect of Boundary Layer Growth on the Stability of Compressible Flows. NASA CR-3474, 1981.

16. Gaponov, S. A., The Influence of Flow Nonparallelism on Disturbance Development in the Supersonic Boundary Layer. Proceedings of the 8th Canadian Congress of Applied Mechanics, pp. 673-674, 1981.

17. Saric, W. S. and Nayfeh, A. H., Nonparallel Stability of Boundary Layers with Pressure Gradients and Suction. AGARD CP-224, 6.1-6.21, 1977.

18. El-Hady, N. M., and Nayfeh, A. H., Nonparallel Stability of Two- Dimensional, Heated Boundary-Layer Flows, In Twelfth Symposium on Naval Hydrodynamics, National Academy of Science, Washington, D.C, 1978.

19. Nayfeh, A. H., Perturbation Methods, Wiley-Interscience, 1973.

20. Van Stijn, T. L. and Van de Vooren, A. I., On the Stability of Almost Parallel Boundary Layer Flows. Comp. \& Fluids, 10, 1983.

21. Herbert, Th. and Bertolotti, F. P., Stability Analysis of Nonparallel Boundary Layer. Bull. Am. Phy. Soc., 32, p 2079, 1987. 
22. Mack, L. M., Review of Linear Compressible Stability Theory. In: Stability for Time Dependent and Spatially varying Flows, ed. D. L. Dwoyer and M. Y. Hussaini, 1985, PP.164187, Springer-Verlag, New York. 
Table 1 Calculated values of different terms in Eq (33) for various Mach numbers at $R=1000$ and the most unstable nonparallel frequency (DIS is the last term in Eq 33).

\begin{tabular}{|l|c|c|c|c|}
\hline$M_{\infty}$ & $-\alpha_{i}$ & $-\left(\alpha_{i}+\varepsilon \tilde{\alpha}_{i}\right)$ & $D I S$ & $\sigma[E q 33]$ \\
\hline \multicolumn{5}{|l|}{ First mode, $\theta=0,0,55,60,65$ respectively } \\
\hline 0.0 & $.6237 e-2$ & $.6813 e-2$ & $-.0421 e-2$ & $.6392 e-2$ \\
0.8 & $.4501 e-2$ & $.4985 e-2$ & $-.0606 e-2$ & $.4378 e-2$ \\
1.6 & $.2626 e-2$ & $.3236 e-2$ & $-.0103 e-2$ & $.3133 e-2$ \\
2.2 & $.2141 e-2$ & $.2843 e-2$ & $-.0072 e-2$ & $.2771 e-2$ \\
3.0 & $.1852 e-2$ & $.2623 e-2$ & $-.0166 e-2$ & $.2458 e-2$ \\
\hline Second mode, $\theta=0$ & \\
\hline 4.5 & $.2878 e-2$ & $.4889 e-2$ & $-.1307 e-2$ & $.3582 e-2$ \\
5.8 & $.3018 e-2$ & $.4687 e-2$ & $-.1349 e-2$ & $.3338 e-2$ \\
7.0 & $.2323 e-2$ & $.3766 e-2$ & $-.1525 e-2$ & $.2241 e-2$ \\
10. & $.6483 e-3$ & $.2443 e-2$ & $-.1315 e-2$ & $.1128 e-2$ \\
\hline
\end{tabular}




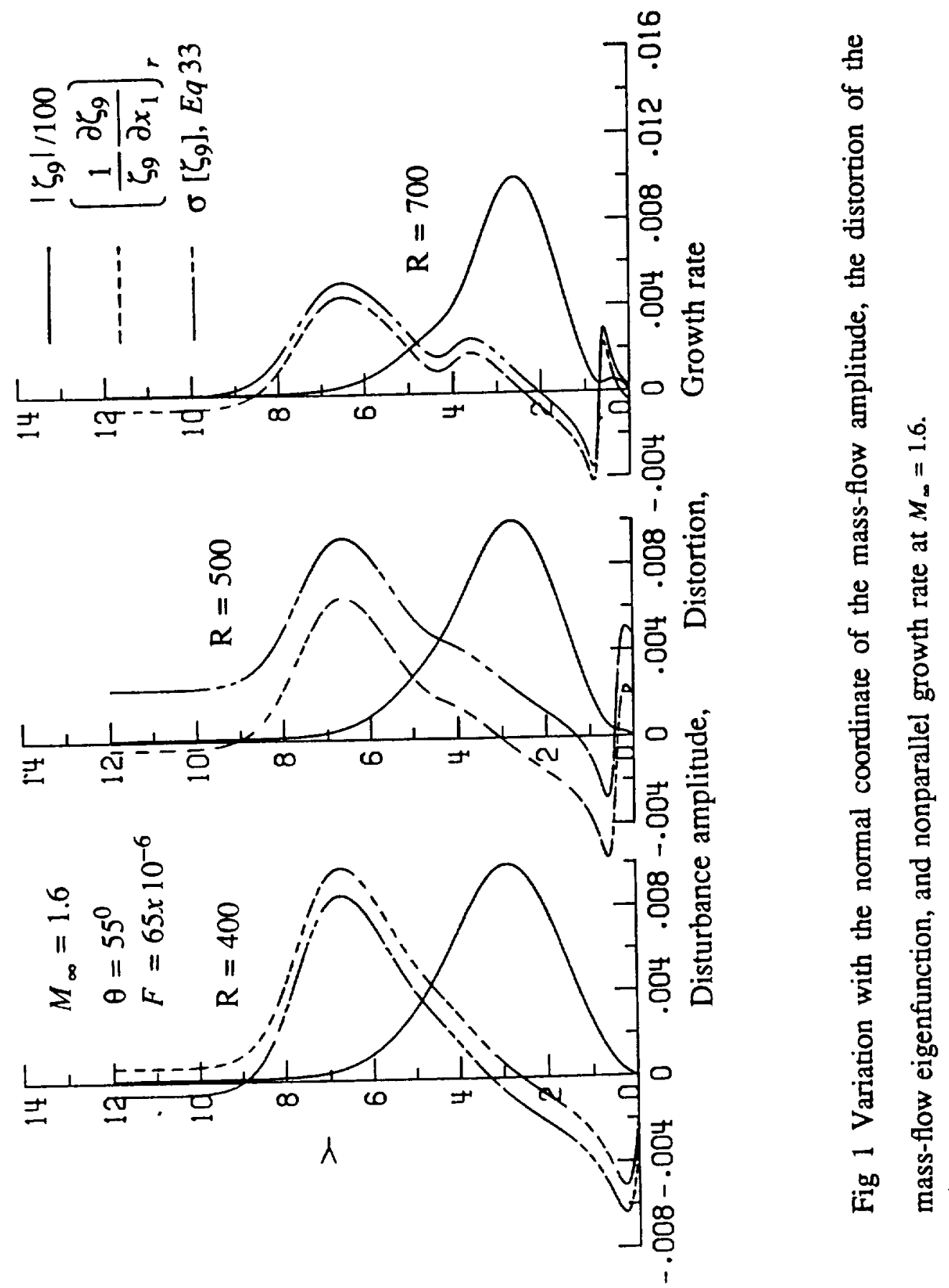




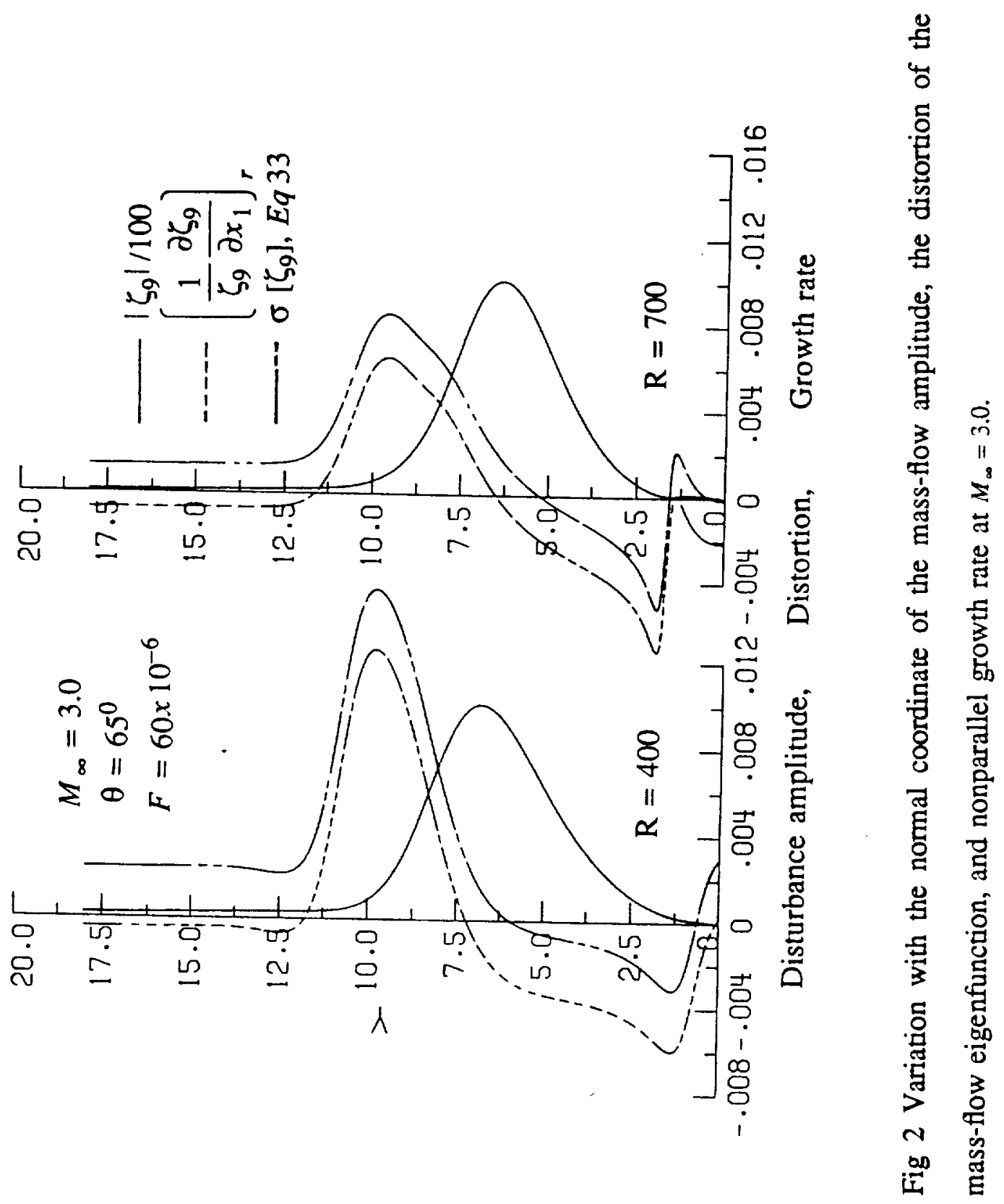




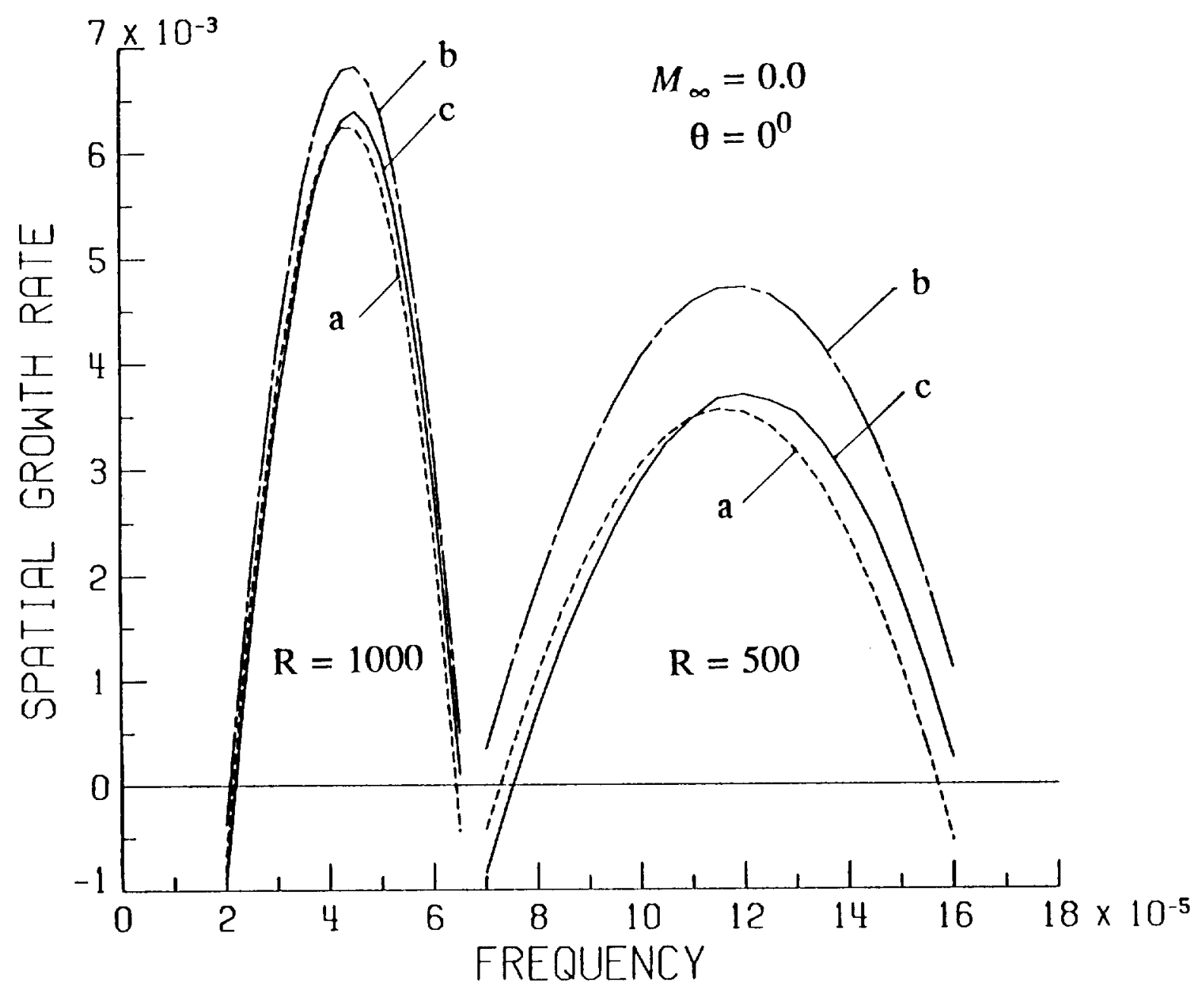

Fig 3a Variation of the spatial growth rates with frequency at subsonic speeds for $2 \mathrm{D}$ wave, [a] $-\alpha_{i},[\mathrm{~b}]-\left(\alpha_{i}+\varepsilon \tilde{\alpha}_{i}\right),[\mathrm{c}]$ Eq 33 based on $\zeta_{q} \max$. 


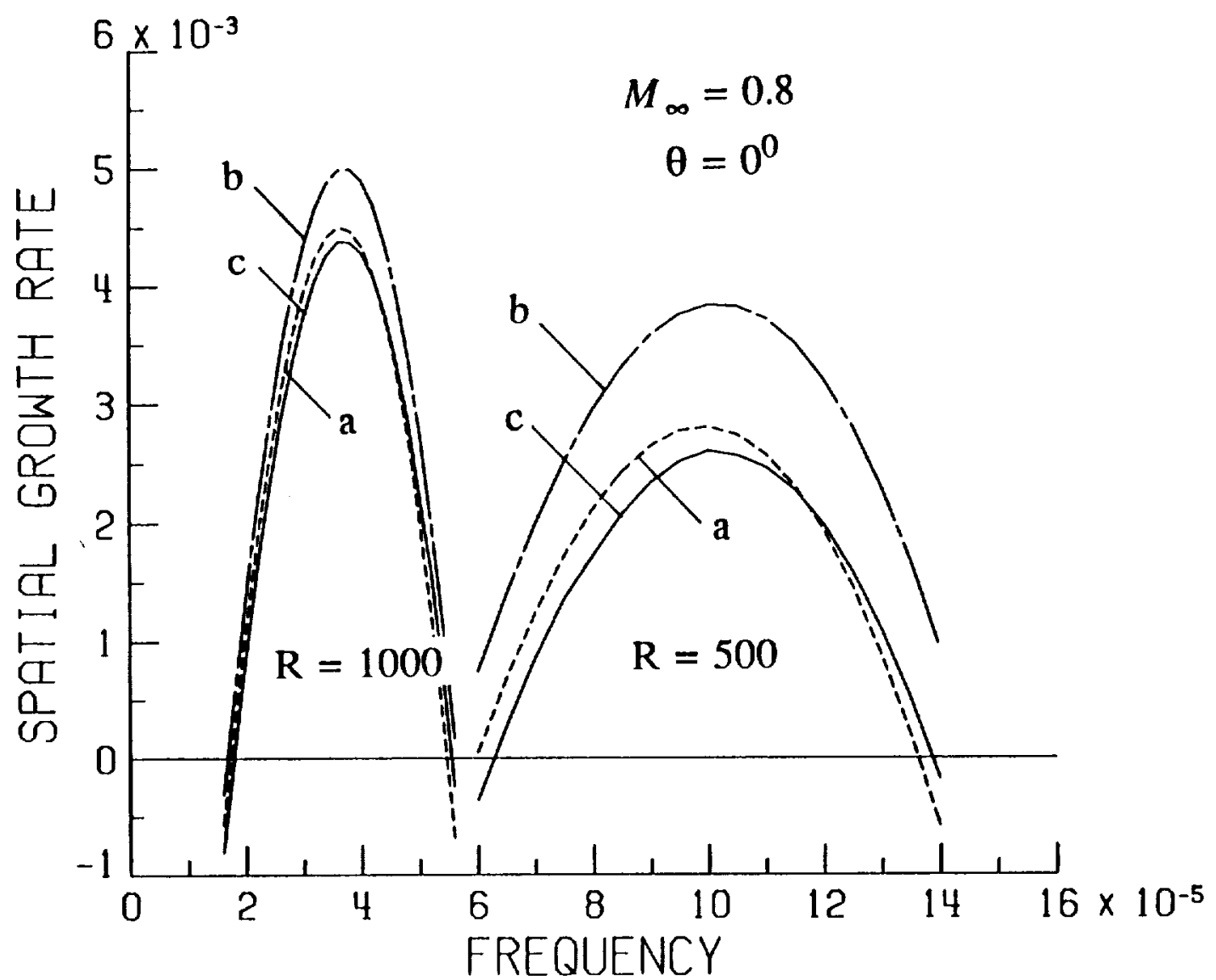

Fig $3 b$ Variation of the spatial growth rates with frequency at subsonic speeds for $2 \mathrm{D}$ wave, [a] $-\alpha_{i},[b]-\left(\alpha_{i}+\varepsilon \alpha_{i}\right),[c]$ Eq 33 based on $\zeta_{9}$ max. 


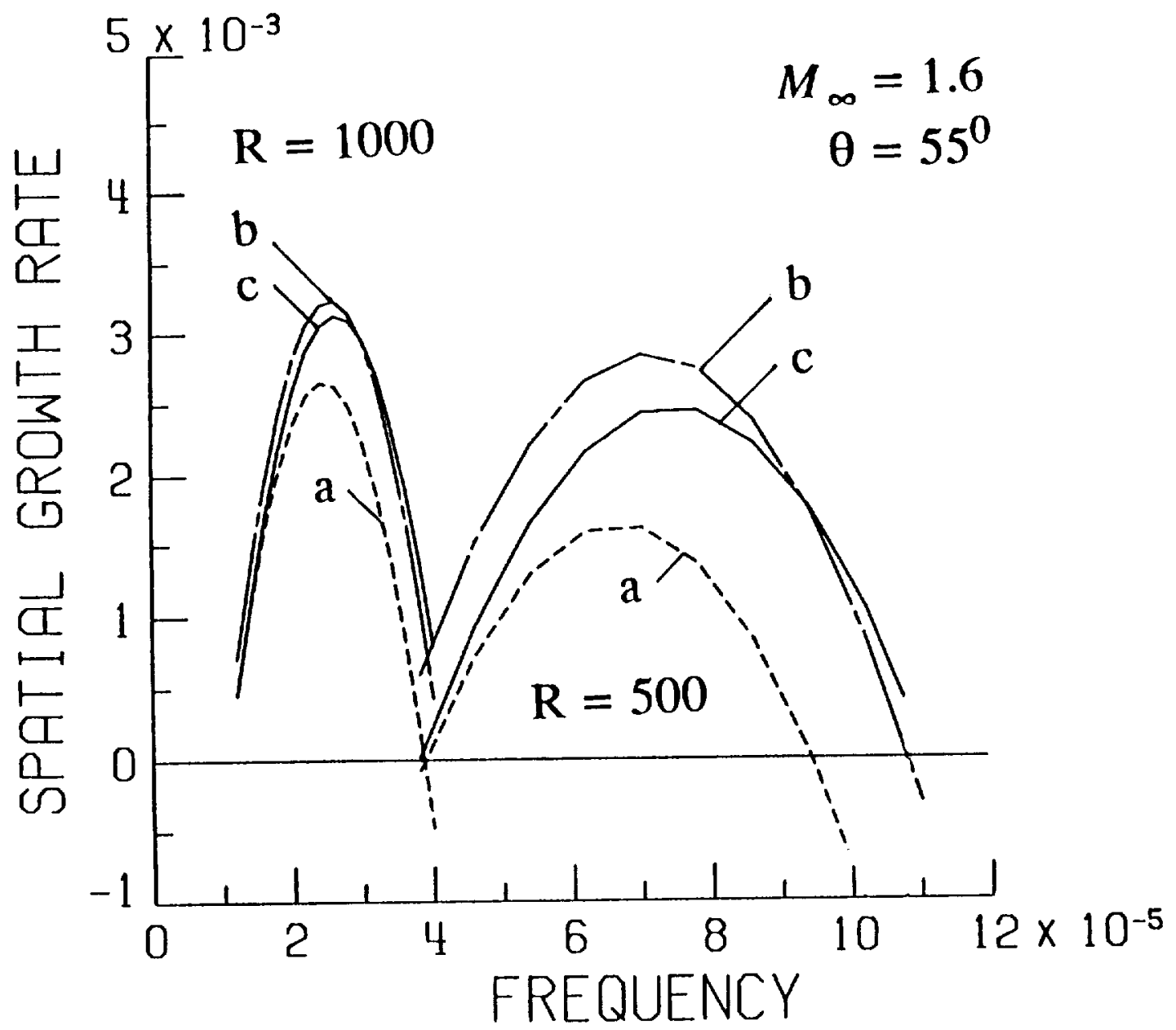

Fig 4a Variation of the spatial growth rates with frequency at supersonic speeds for a first mode oblique wave, [a] $-\alpha_{1},[\mathrm{~b}]-\left(\alpha_{1}+\varepsilon \alpha_{1}\right)$, [c] Eq 33 based on $\zeta_{9}$ max. 


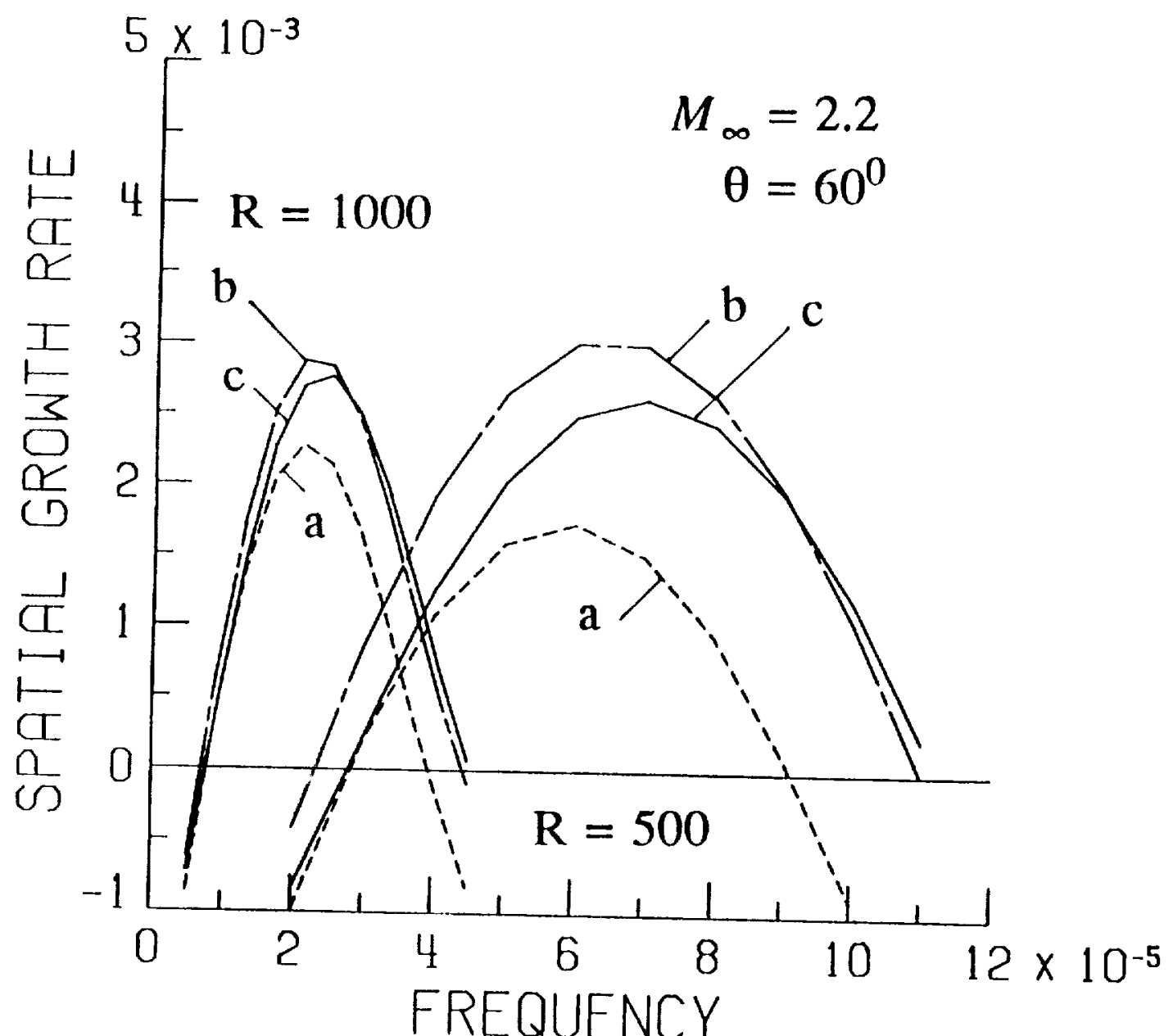

Fig $4 \mathrm{~b}$ Variation of the spatial growth rates with frequency at supersonic speeds for a first mode oblique wave, [a] $-\alpha_{i}$, [b] $-\left(\alpha_{i}+\varepsilon \alpha_{i}\right)$, [c] Eq 33 based on $\zeta_{9} \max$. 


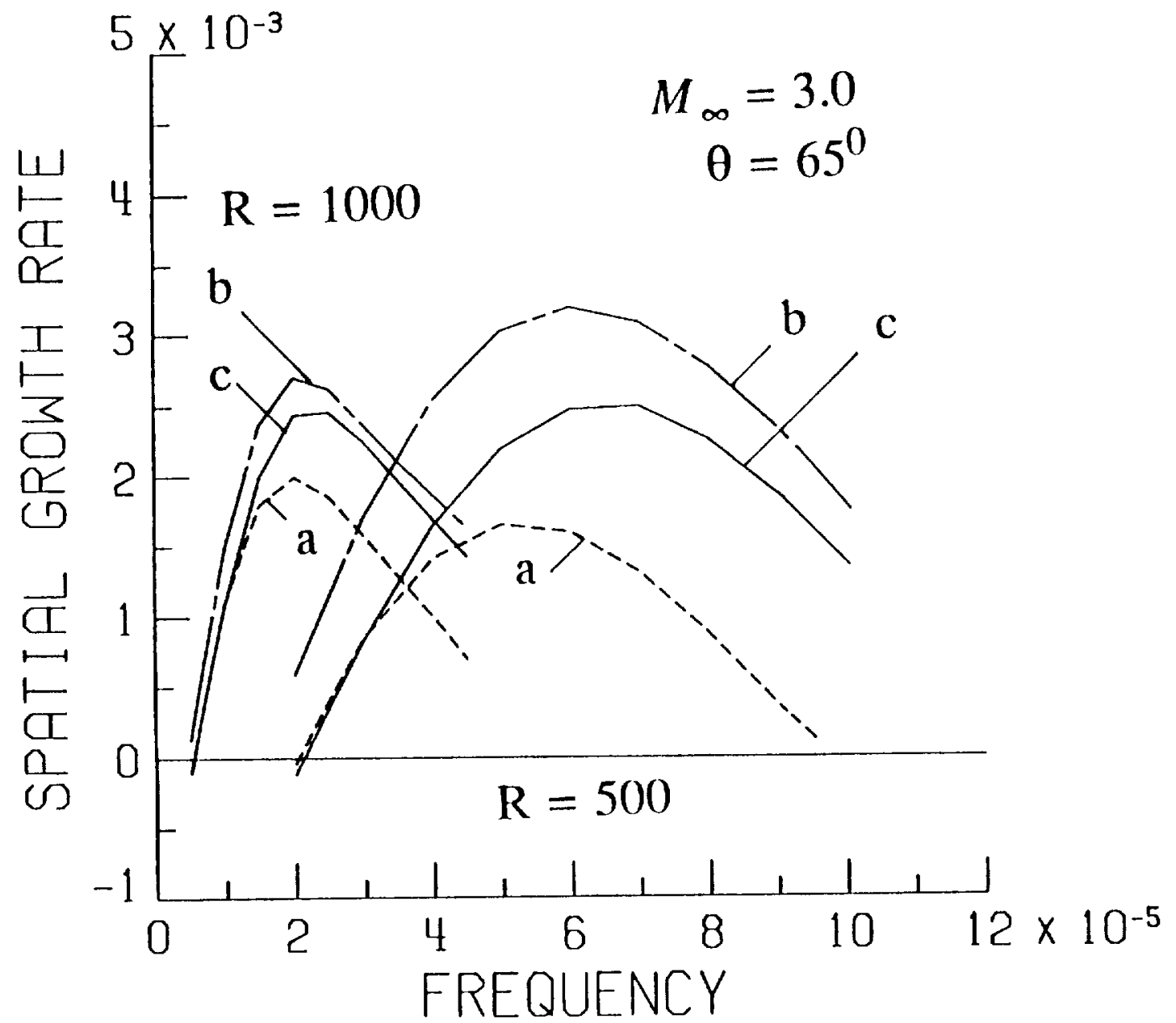

Fig $4 \mathrm{c}$ Variation of the spatial growth rates with frequency at supersonic speeds for a first mode oblique wave, [a] $-\alpha_{i},[b]-\left(\alpha_{i}+\varepsilon \pi_{i}\right)$, [c] Eq 33 based on $\zeta_{9}$ max. 


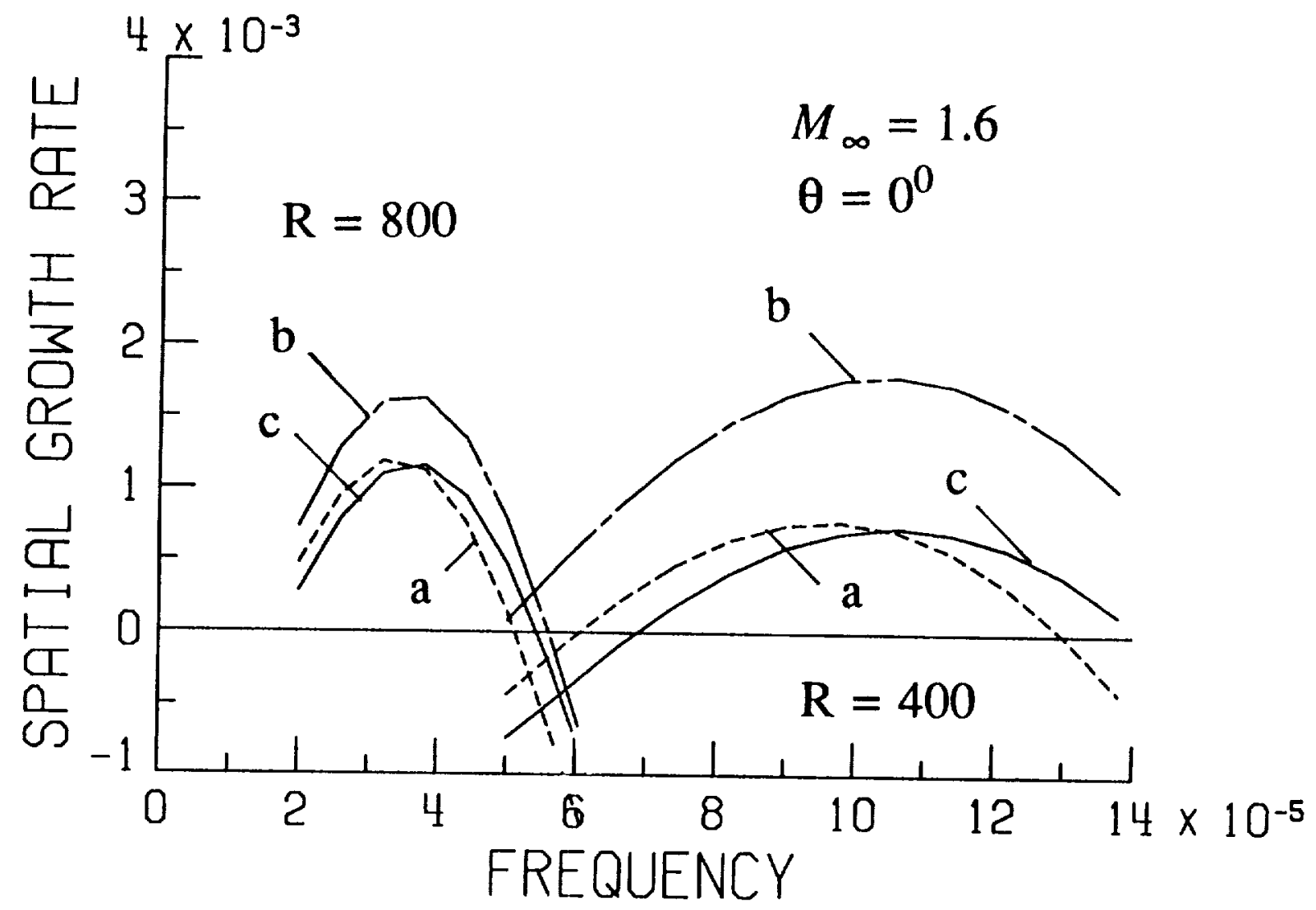

Fig 5 Variation of the spatial growth rates with frequency at supersonic speeds for a $2 \mathrm{D}$ wave, [a] $-\alpha_{i},[\mathrm{~b}]-\left(\alpha_{i}+\varepsilon \alpha_{i}\right)$, [c] Eq 33 based on $\zeta_{9} \max$. 


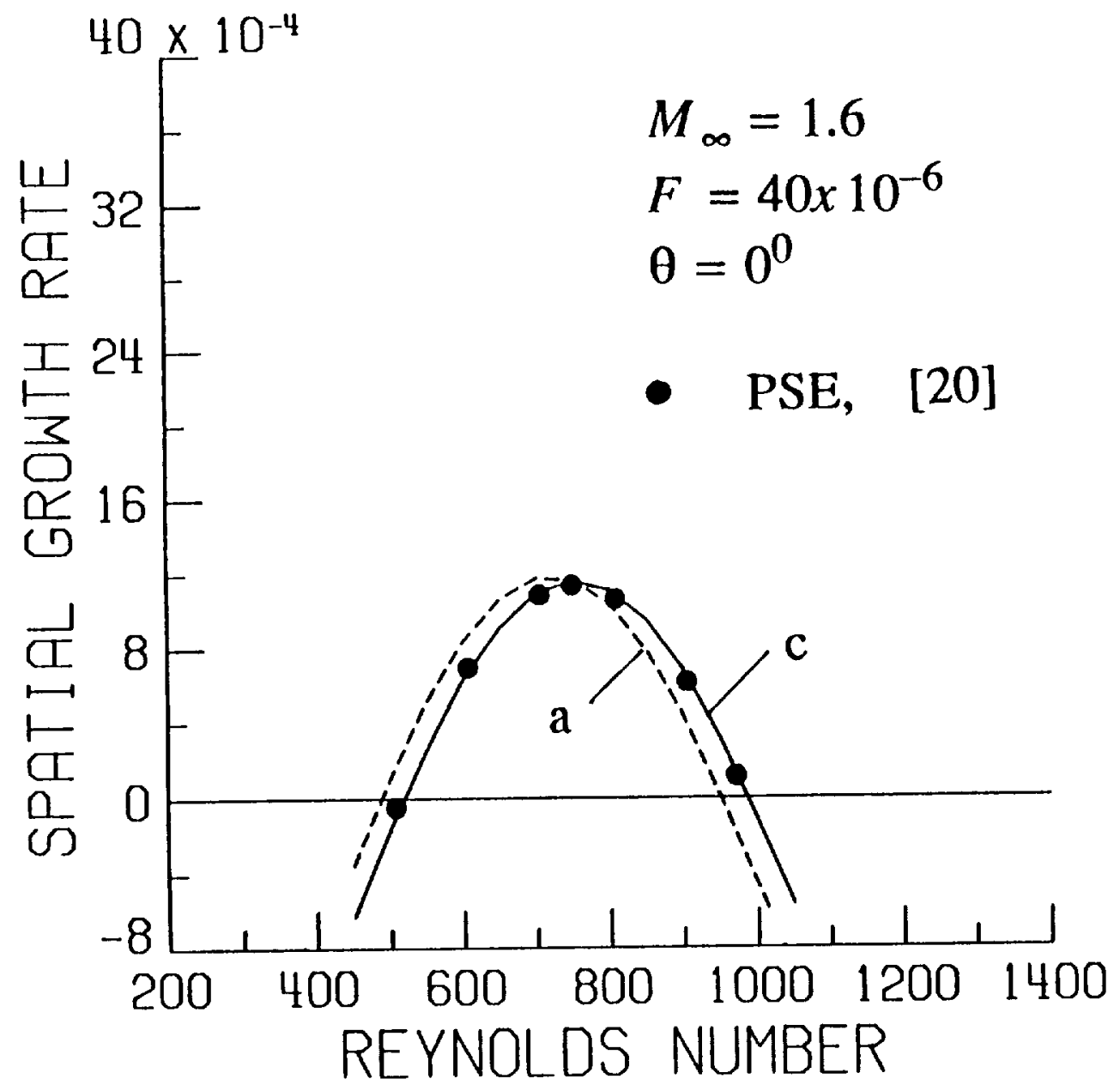

Fig 6a Variation of the spatial growth rates with Reynolds number for a $2 \mathrm{D}$ wave at $M_{-}=1.6$, and comparison with PSE results. [a] $-\alpha_{i}$, [c] Eq 33 based on $\zeta$ gax. 


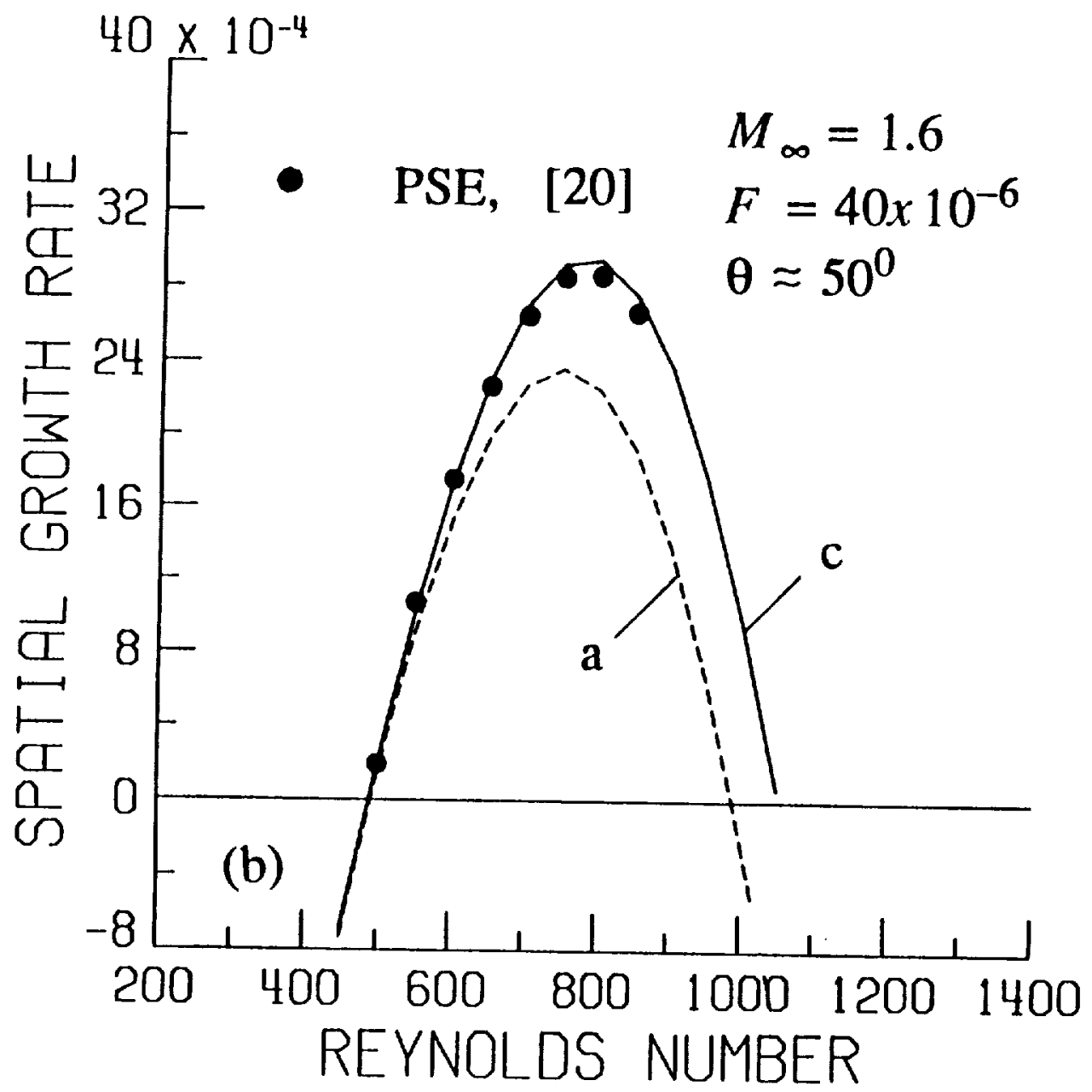

Fig $6 \mathrm{~b}$ Variation of the spatial growth rates with Reynolds number for an oblique wave at $M_{\infty}=1.6$, and comparison with PSE results. [a] $-\alpha_{i}$, [c] Eq 33 based on $\zeta_{9} \max$. 


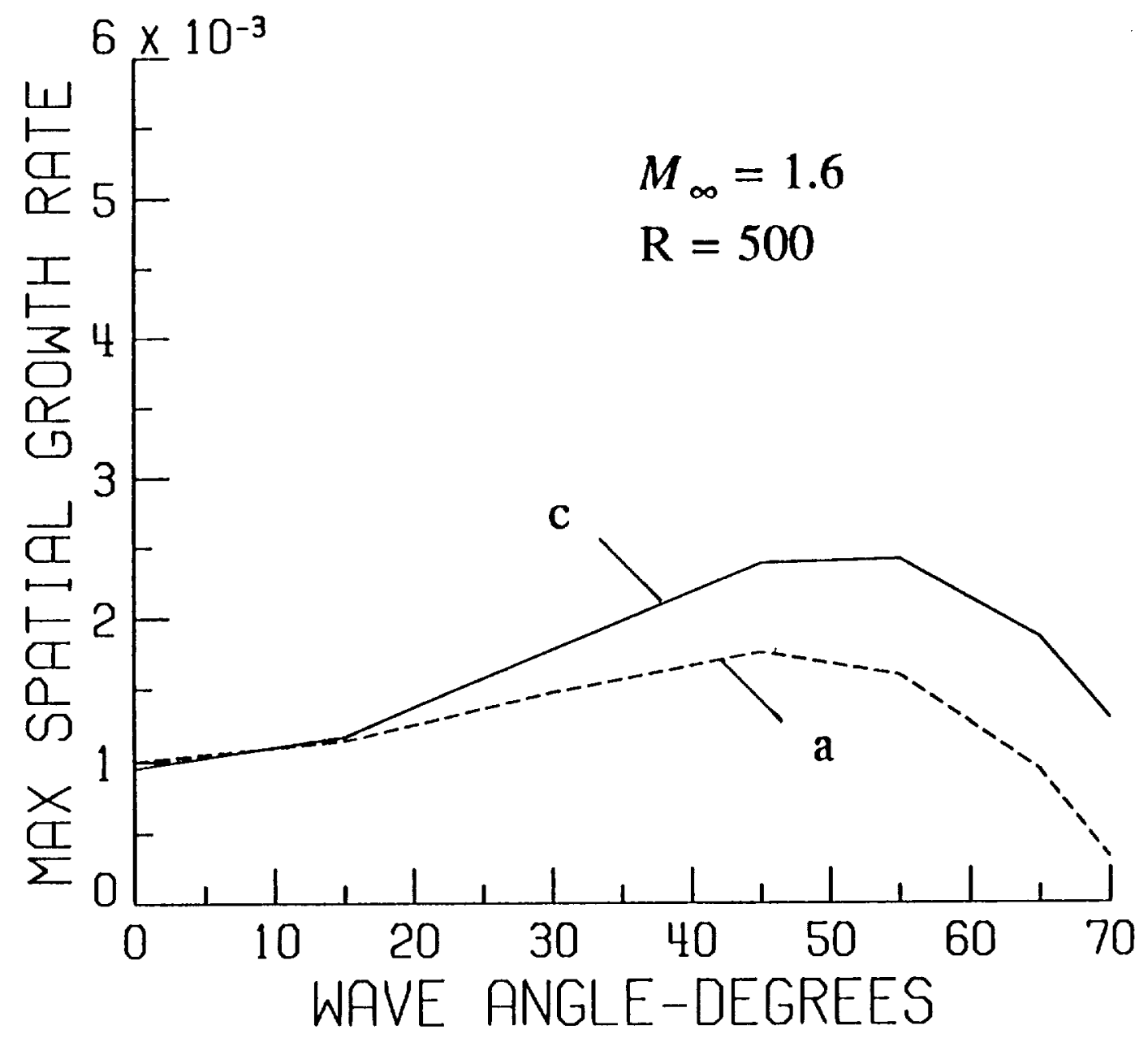

Fig 7 Effect of wave obliqueness on the maximum growth rate (w.r.t. frequency) at $M_{\infty}=1.6$, [a] $-\alpha_{i}$, [c] Eq 33 based on $\zeta_{9} \max$. 


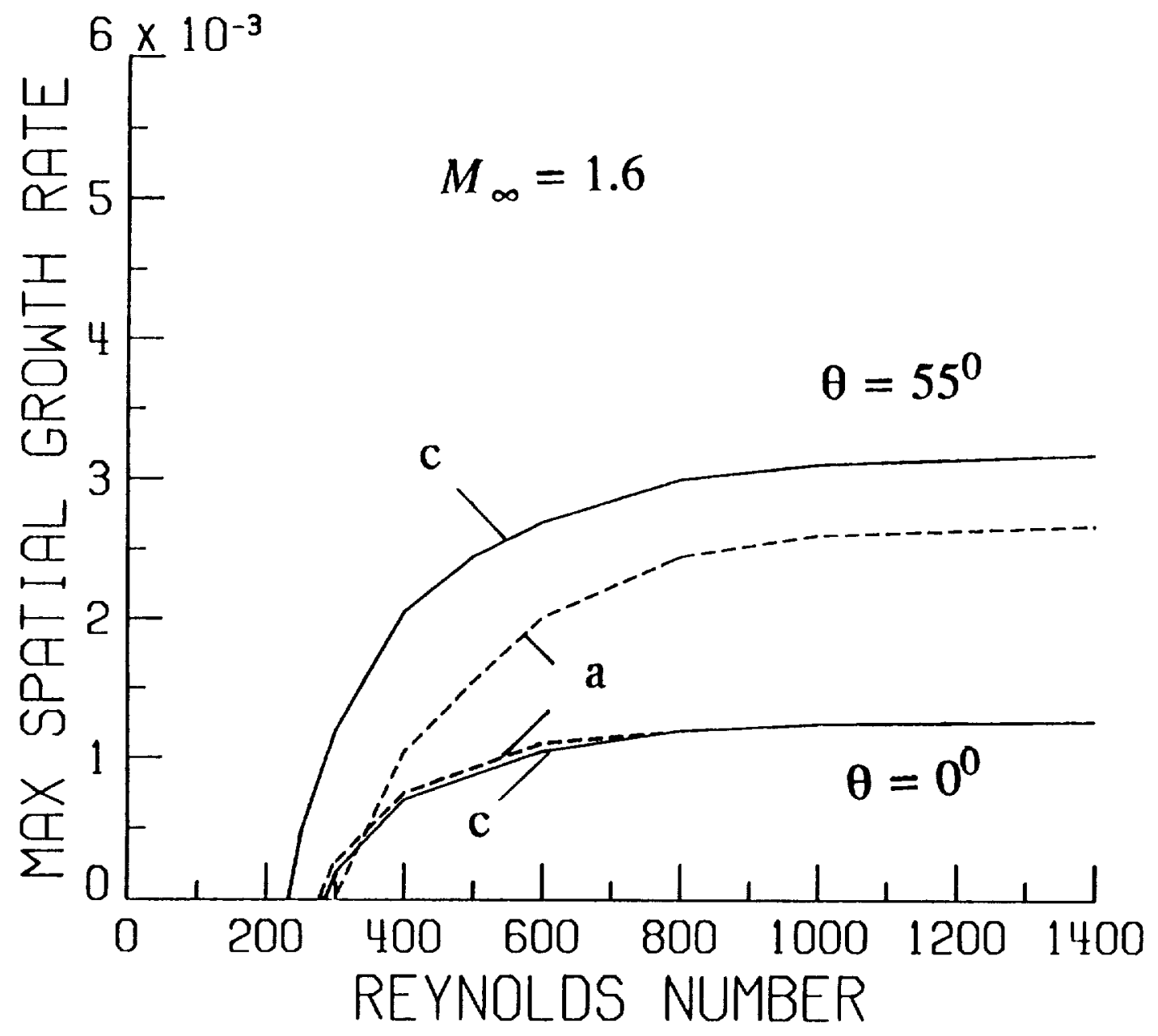

Fig 8 Variation of maximum spatial growth rates (w.r.t. frequency) with Reynolds number for 2D and oblique wave at $M_{-}=1.6$, [a] $-\alpha_{i}$, [c] Eq 33 based on $\zeta_{9}$ max. 


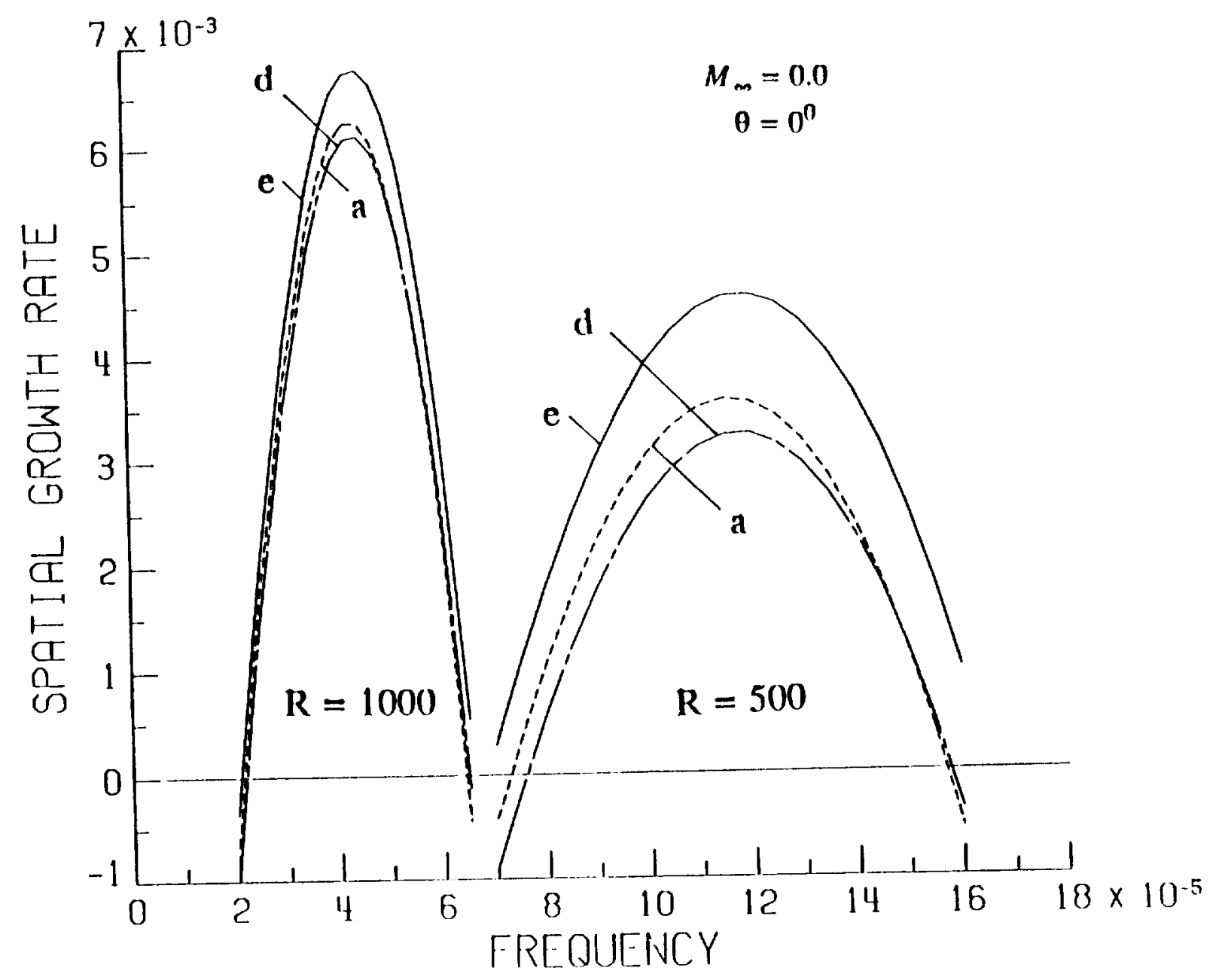

Fig 9a Variation of the spatial growth rate with frequency for a $2 \mathrm{D}$ wave at $M_{\infty}=0,[\mathrm{a}]-\alpha_{i},[\mathrm{~d}]$ Eq 33 based on $\hat{E}$, [e] Eq 33 based on $E$. 


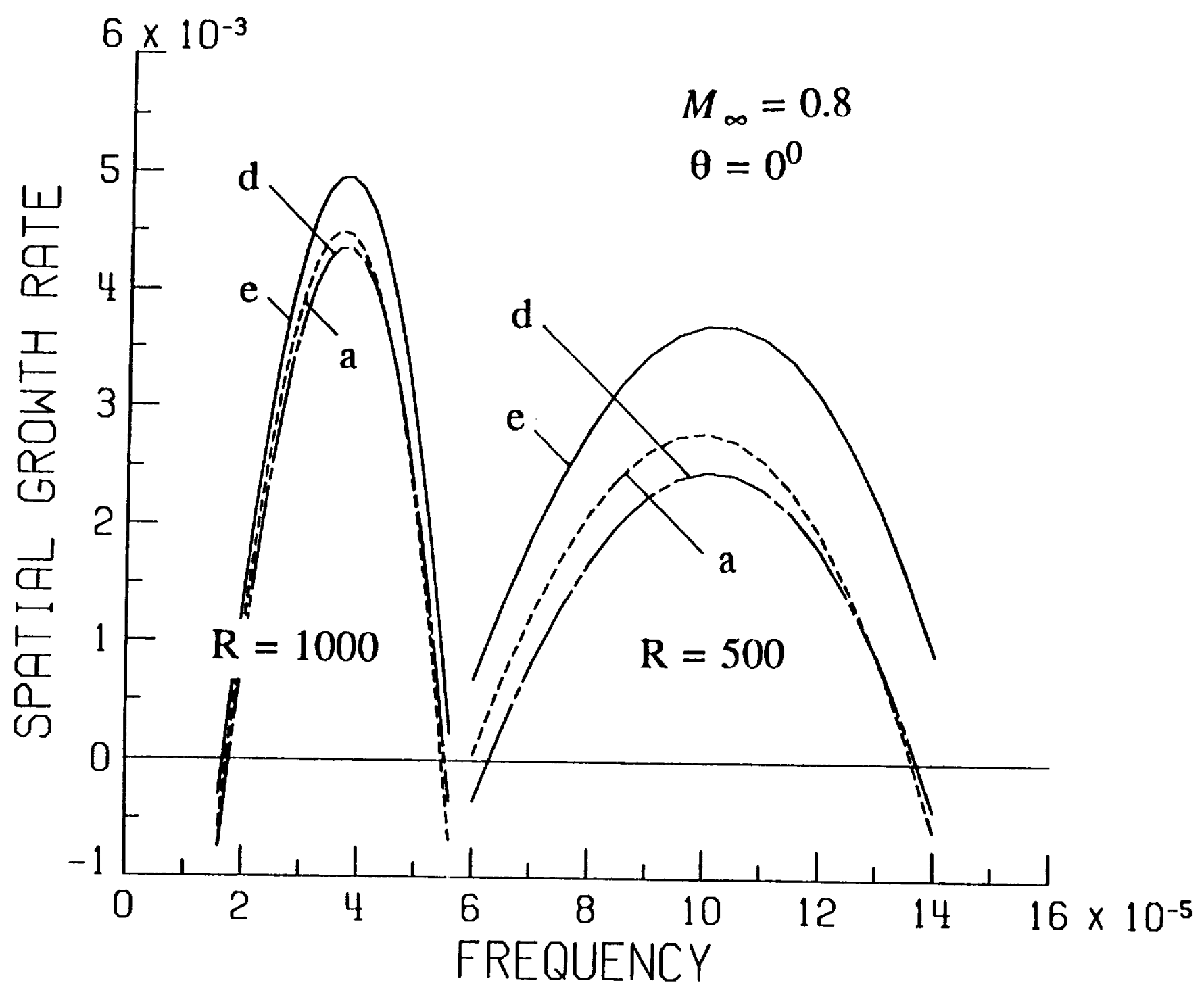

Fig $9 \mathrm{~b}$ Variation of the spatial growth rate with frequency for a 2D wave at $M_{-}=0.8$, [a] $-\alpha_{i}$, [d] Eq 33 based on $\hat{E}$, [e] Eq 33 based on $E$. 


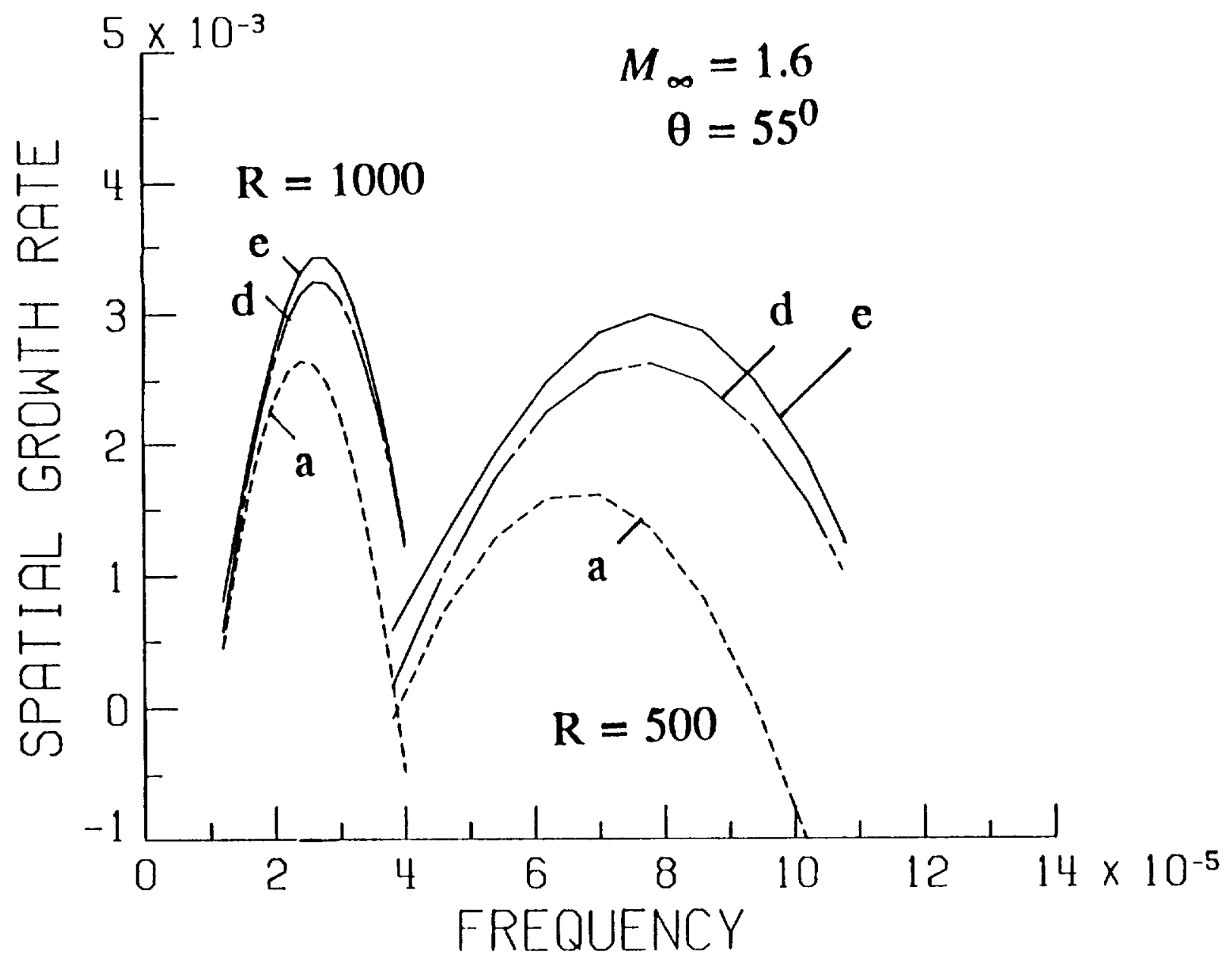

Fig 10a Variation of the spatial growth rate with frequency for a first mode oblique wave at $M_{-}=1.6,[\mathrm{a}]-\alpha_{i}$, [d] Eq 33 based on $\hat{E},[\mathrm{e}] \mathrm{Eq} 33$ based on $E$. 


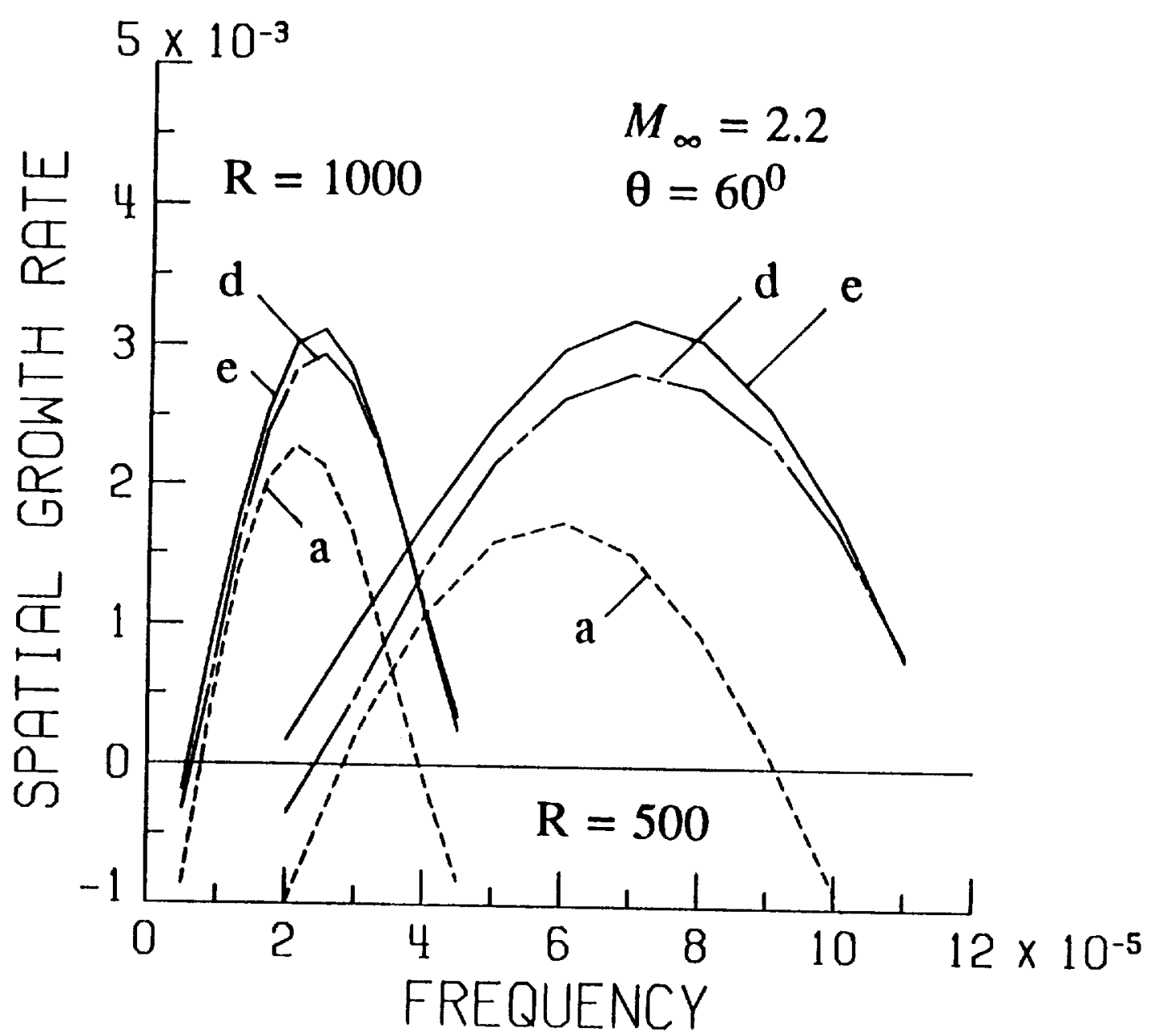

Fig $10 \mathrm{~b}$ Variation of the spatial growth rate with frequency for a first mode oblique wave at $M_{\infty}=2.2,[\mathrm{a}]-\alpha_{i}$, [d] Eq 33 based on $\hat{E}$, [e] Eq 33 based on $E$. 


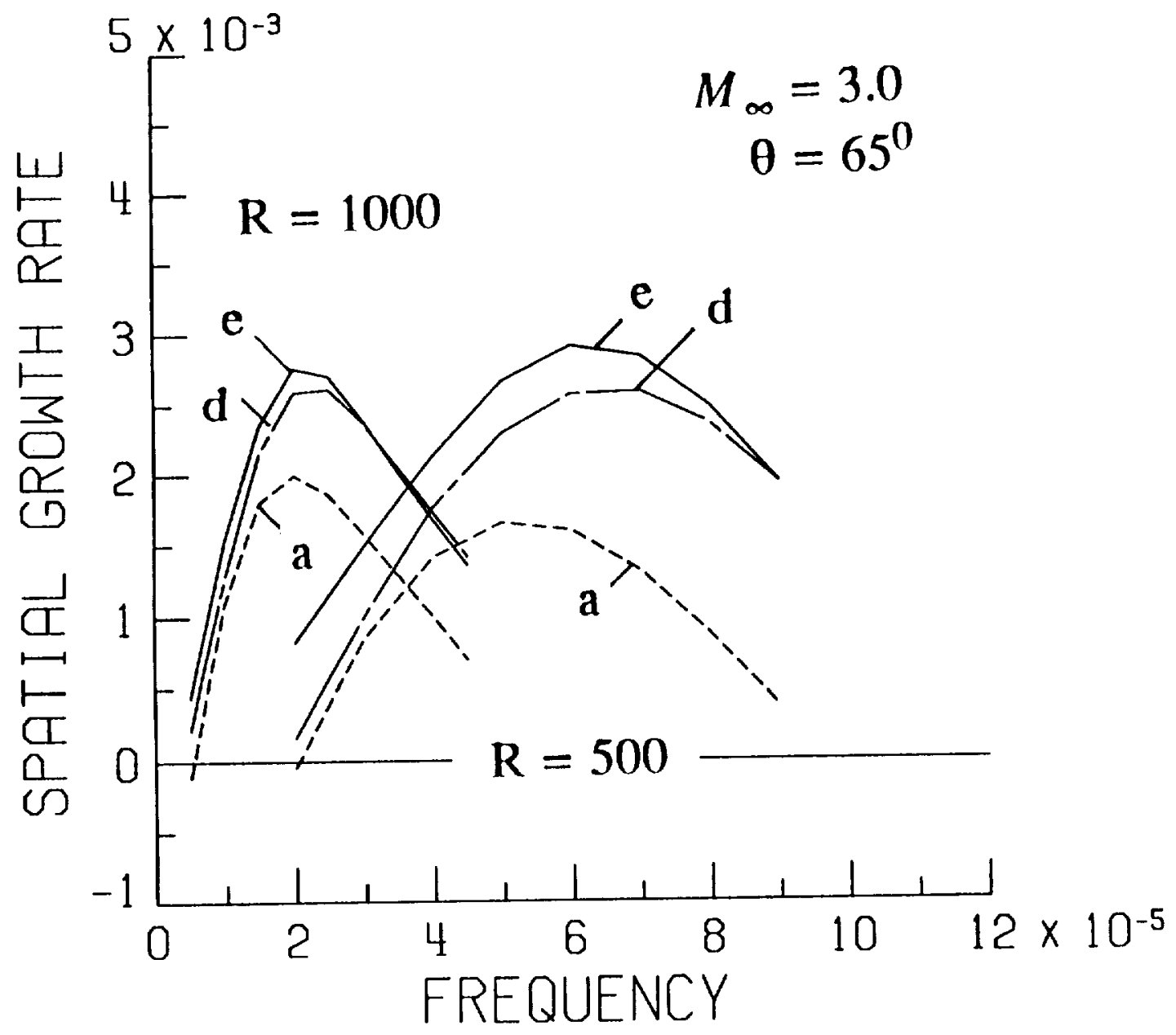

Fig $10 \mathrm{c}$ Variation of the spatial growth rate with frequency for a first mode oblique wave at $M_{\infty}=3.0$, [a] $-\alpha_{i}$, [d] Eq 33 based on $\hat{E}$, [e] Eq 33 based on $E$. 


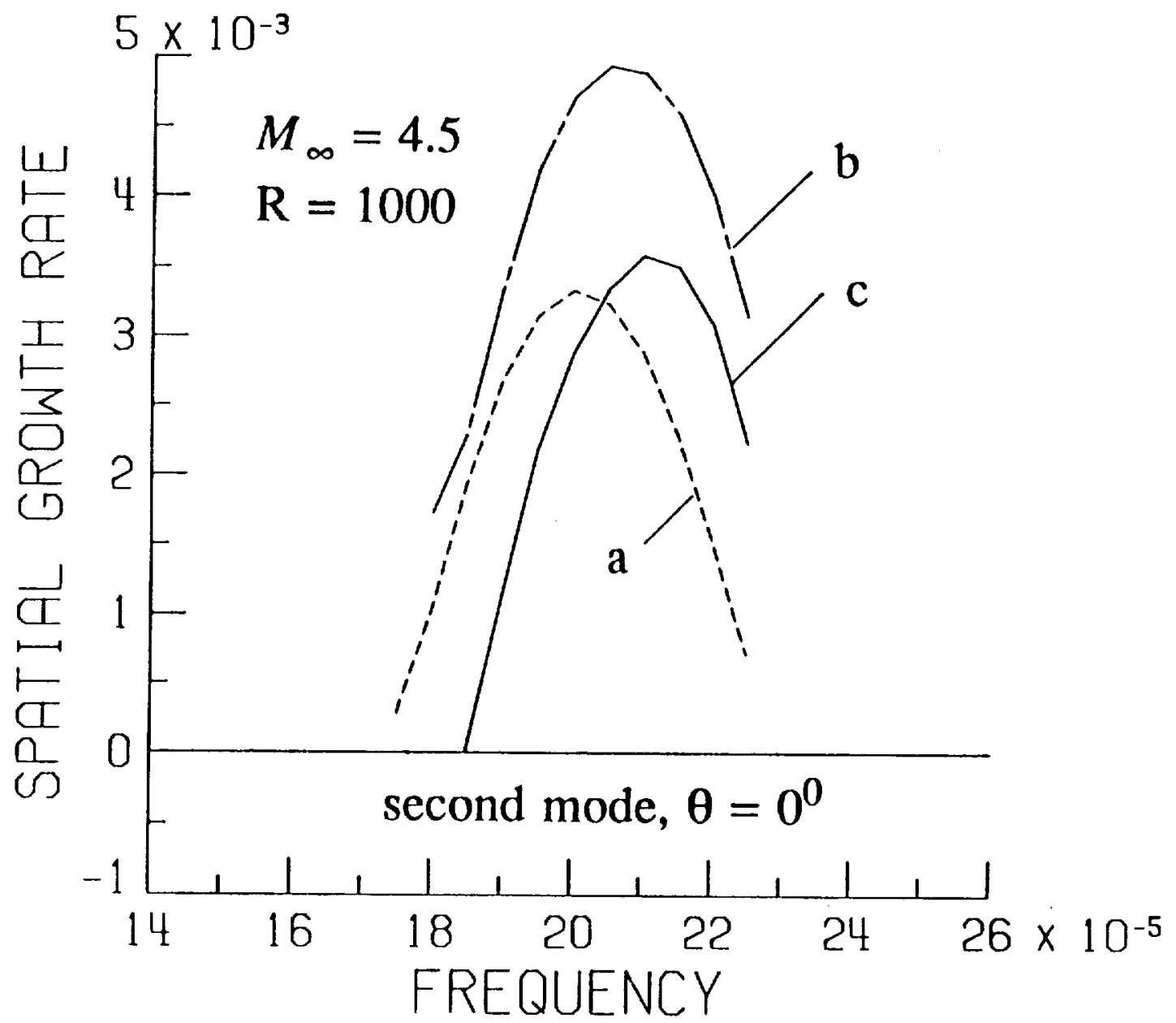

Fig 11a Variation of the spatial growth rate with frequency for a 2D second mode at $R=1000$ and $M_{\infty}=4.5$. [a] $-\alpha_{i},[\mathrm{~b}]-\left(\alpha_{i}+\varepsilon \tilde{x}_{i}\right),[\mathrm{c}] \mathrm{Eq} 33$ based on $\zeta_{9} \max$. 


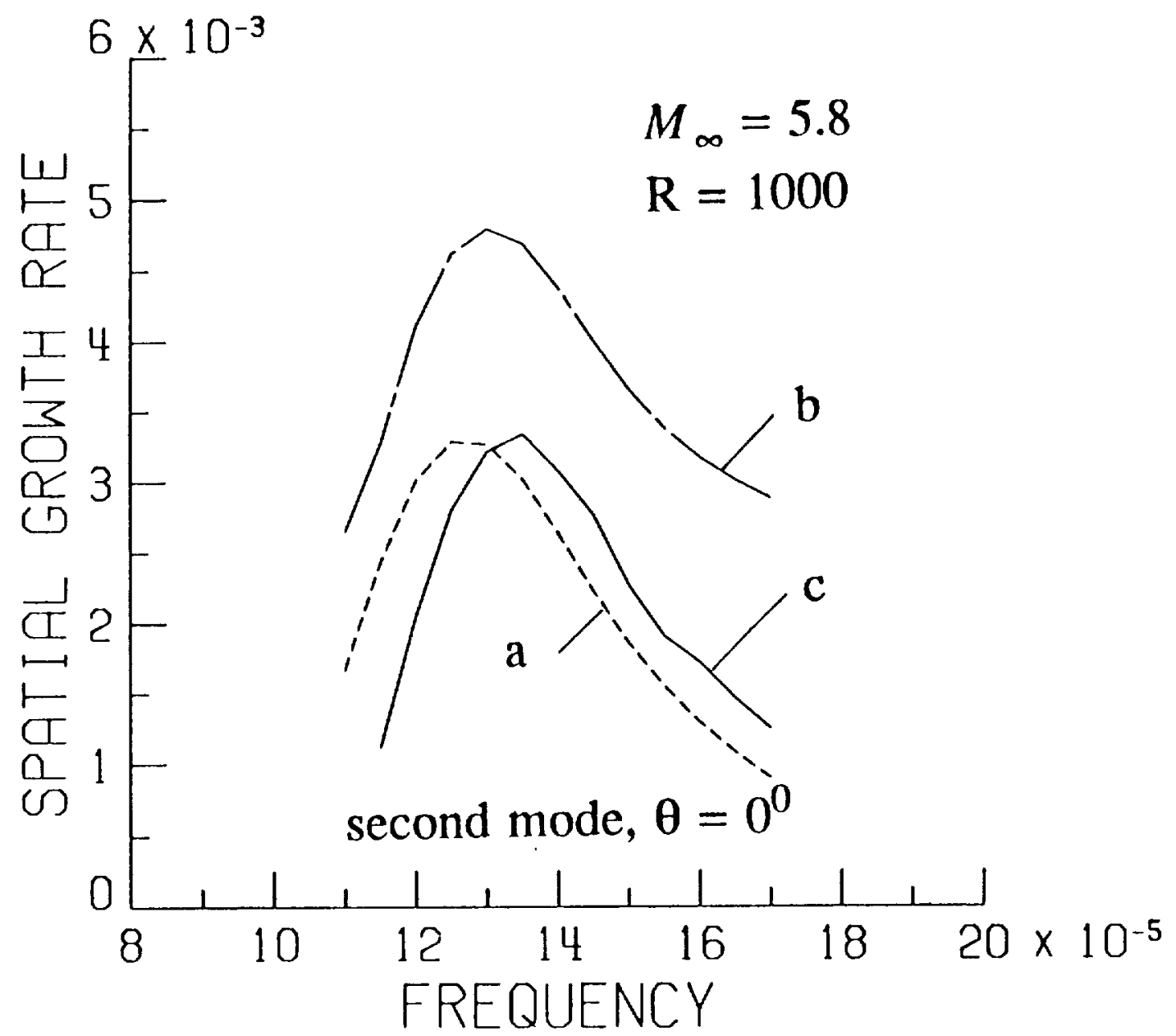

Fig $11 \mathrm{~b}$ Variation of the spatial growth rate with frequency for a $2 \mathrm{D}$ second mode at $R=1000$ and $M_{-}=5.8$. [a] $-\alpha_{i},[\mathrm{~b}]-\left(\alpha_{i}+\varepsilon \tilde{\alpha}_{i}\right)$, [c] Eq 33 based on $\zeta_{9} \max$. 


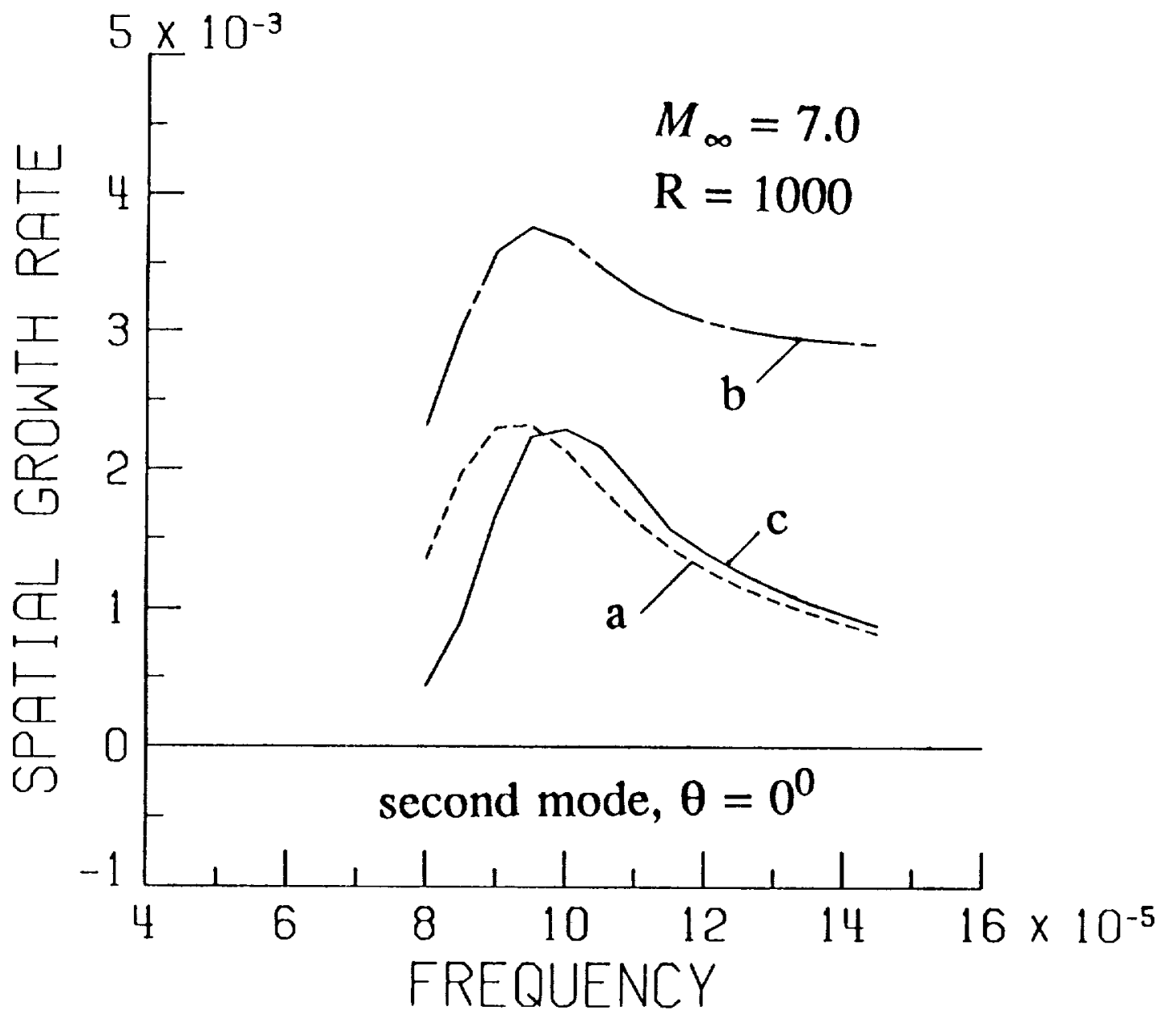

Fig 11c Variation of the spatial growth rate with frequency for a 2D second mode at $R=1000$ and $M_{-}=$7.0. [a] $-\alpha_{i},[b]-\left(\alpha_{i}+\varepsilon \alpha_{i}\right)$, [c] Eq 33 based on $\zeta_{9} \max$. 


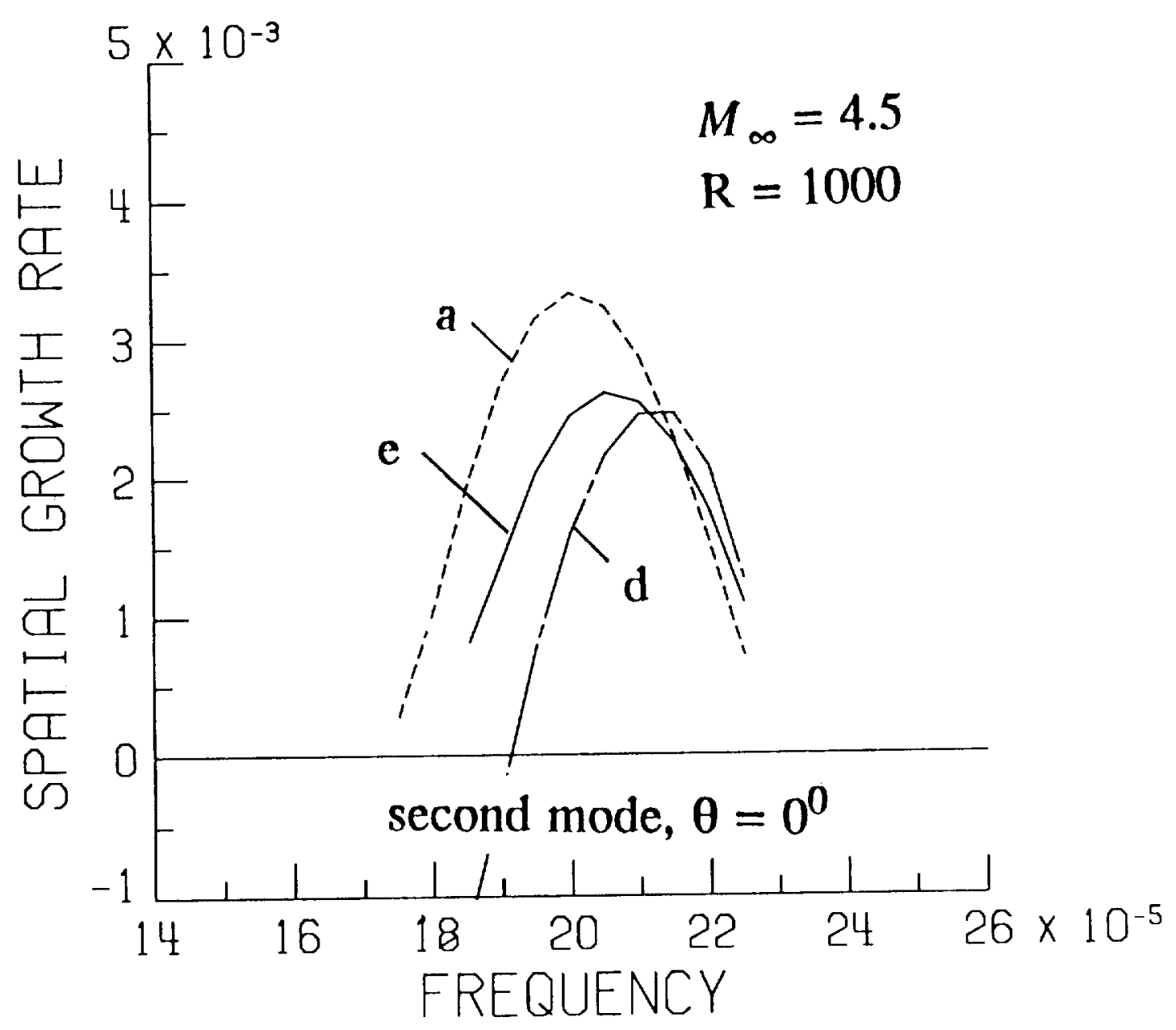

Fig 12a Variation of the spatial growth rate with frequency for a $2 \mathrm{D}$ second mode at $M_{\infty}=4.5$ and $R=1000$, [a] $-\alpha_{i},[\mathrm{~d}]$ Eq 33 based on $\hat{E}$, [e] Eq 33 based on $E$. 


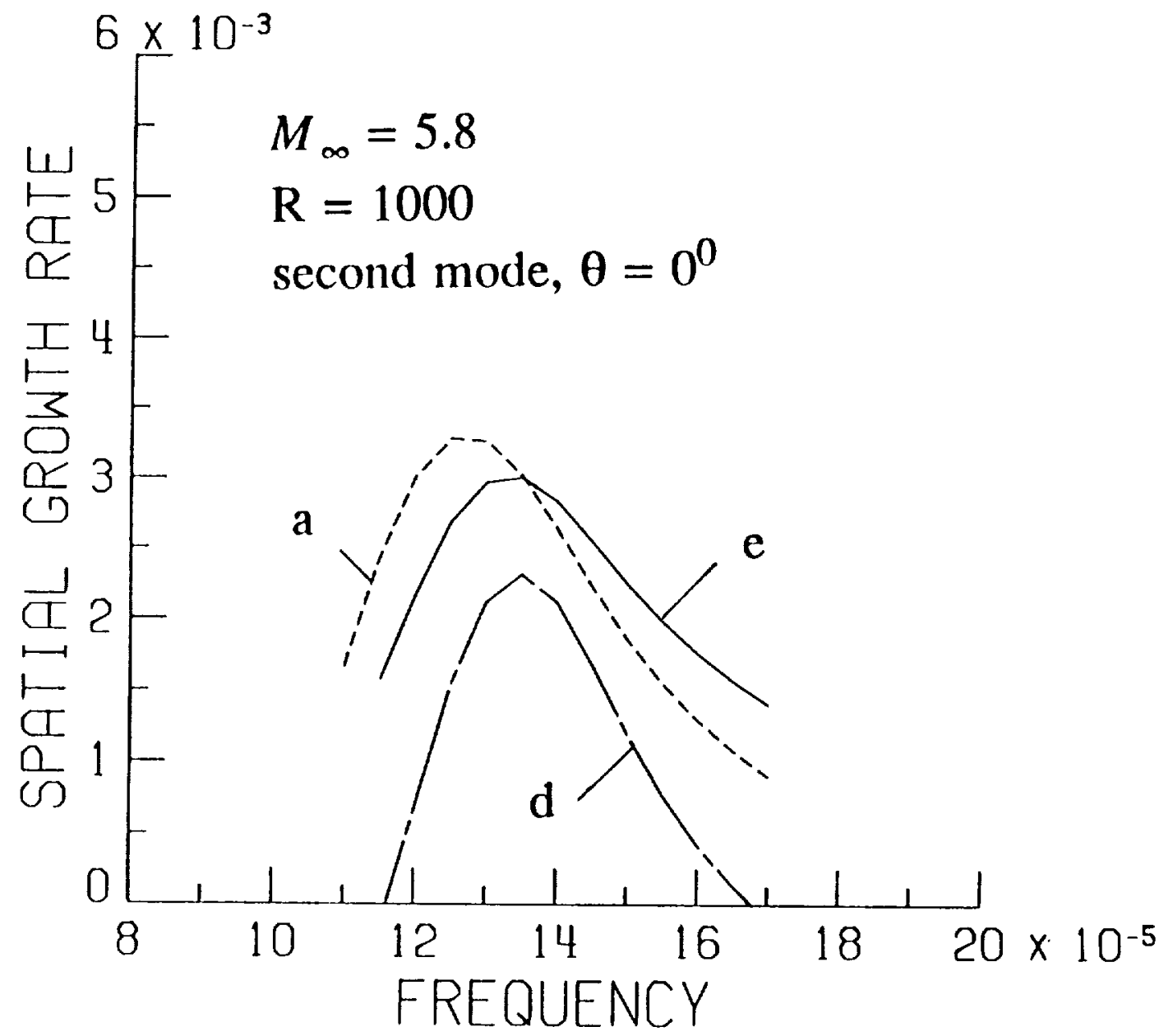

Fig $12 \mathrm{~b}$ Variation of the spatial growth rate with frequency for a $2 \mathrm{D}$ second mode at $M_{-}=5.8$ and $R=1000,[a]-a_{i}$, [d] Eq 33 based on $\hat{E}$, [e] Eq 33 based on $E$. 


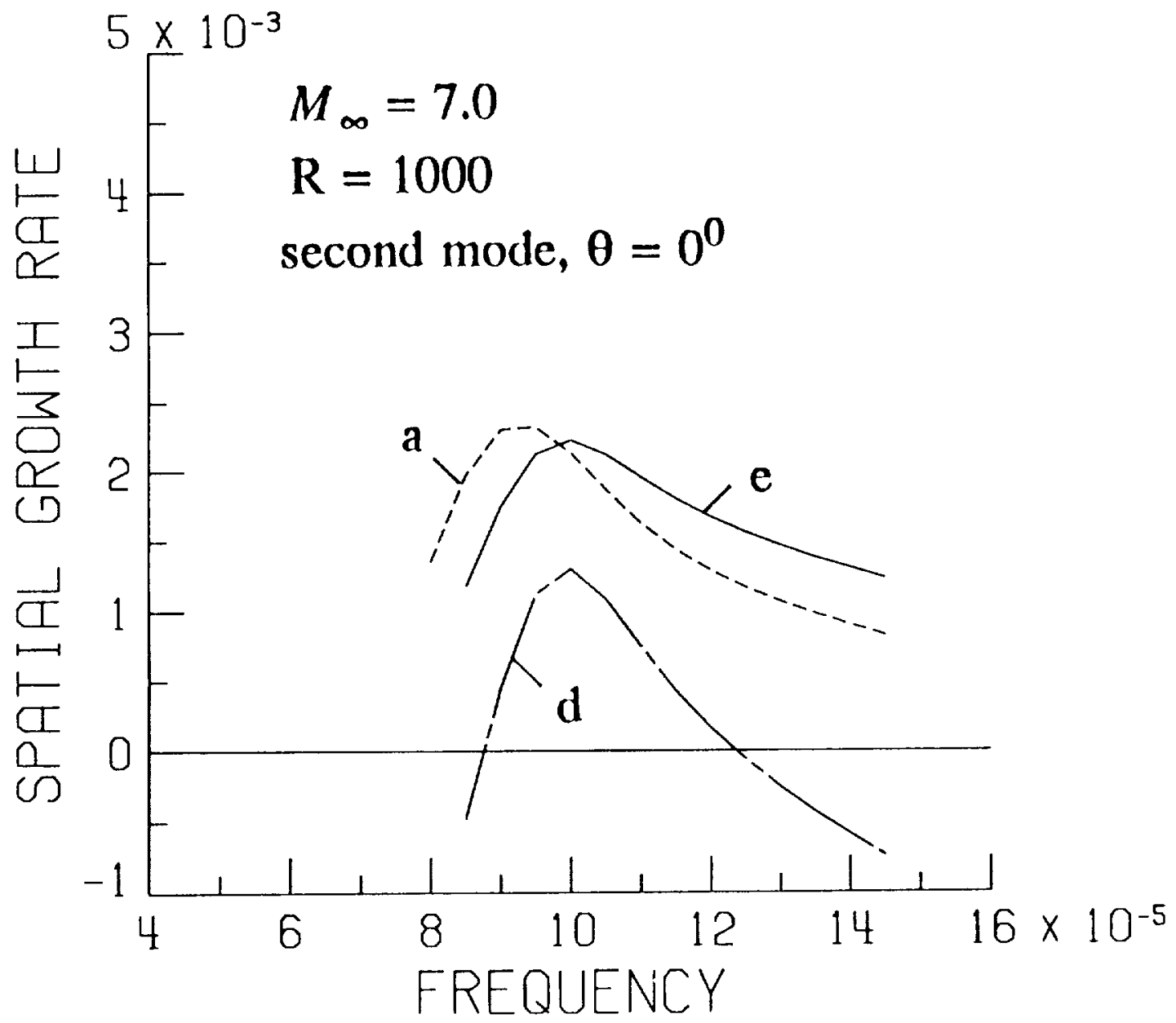

Fig 12c Variation of the spatial growth rate with frequency for a $2 \mathrm{D}$ second mode at $M_{\infty}=7.0$ and $R=1000$, [a] $-\alpha_{i}$, [d] Eq 33 based on $\hat{E}$, [e] Eq 33 based on $E$. 


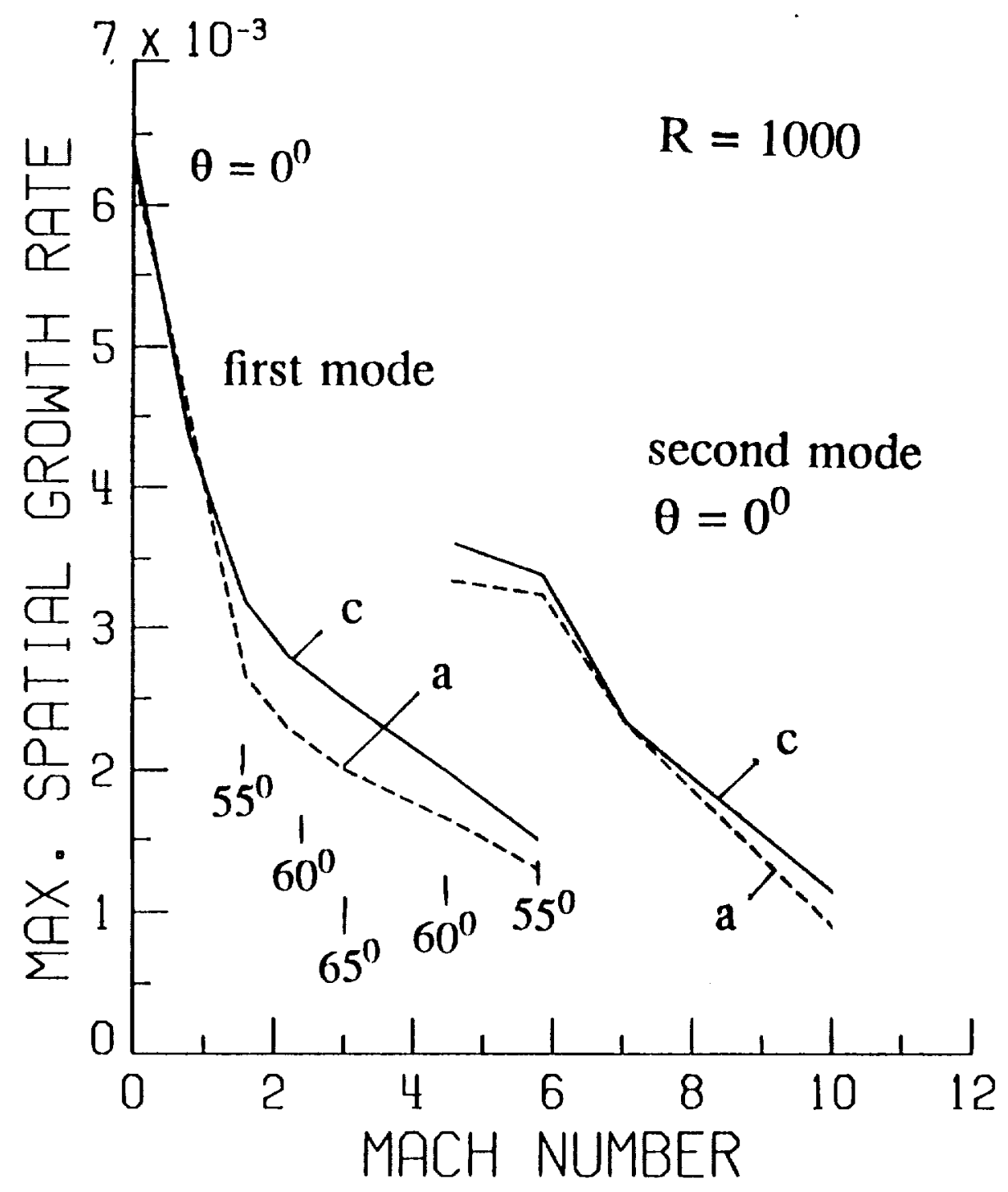

Fig 13a Effect of flow nonparallelism on the maximum spatial growth rates at $R=1000$, and for a range of Mach numbers from 0 to 10, [a] $-\alpha_{i}$, [c] Eq 33 based on $\zeta_{9}$ max. 


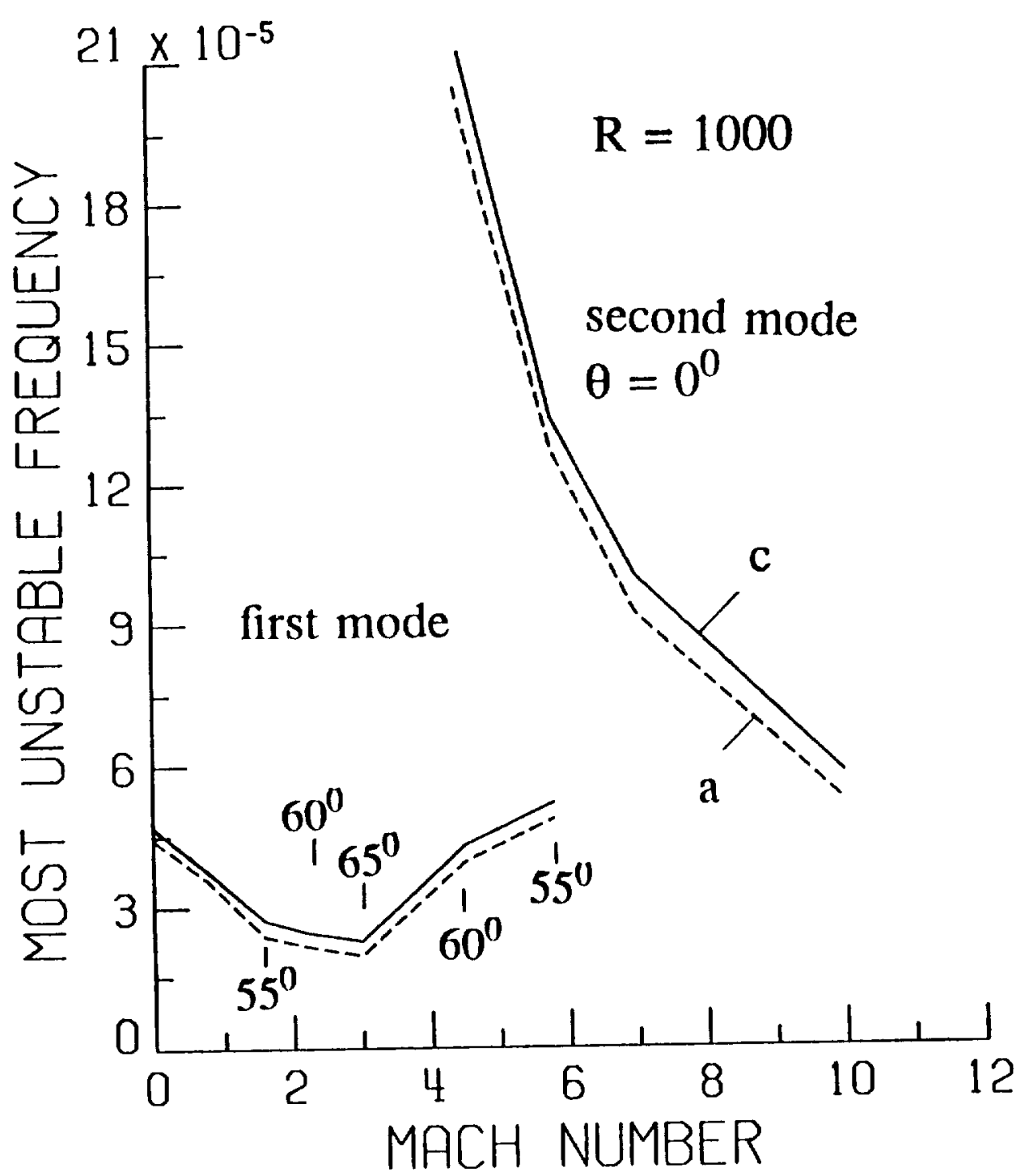

Fig 13b Effect of flow nonparallelism on the most unstable frequencies at $R=1000$, and for a range of Mach numbers from 0 to 10 , [a] $-\alpha_{i}$, [c] Eq 33 based on $\zeta_{9}$ max. 


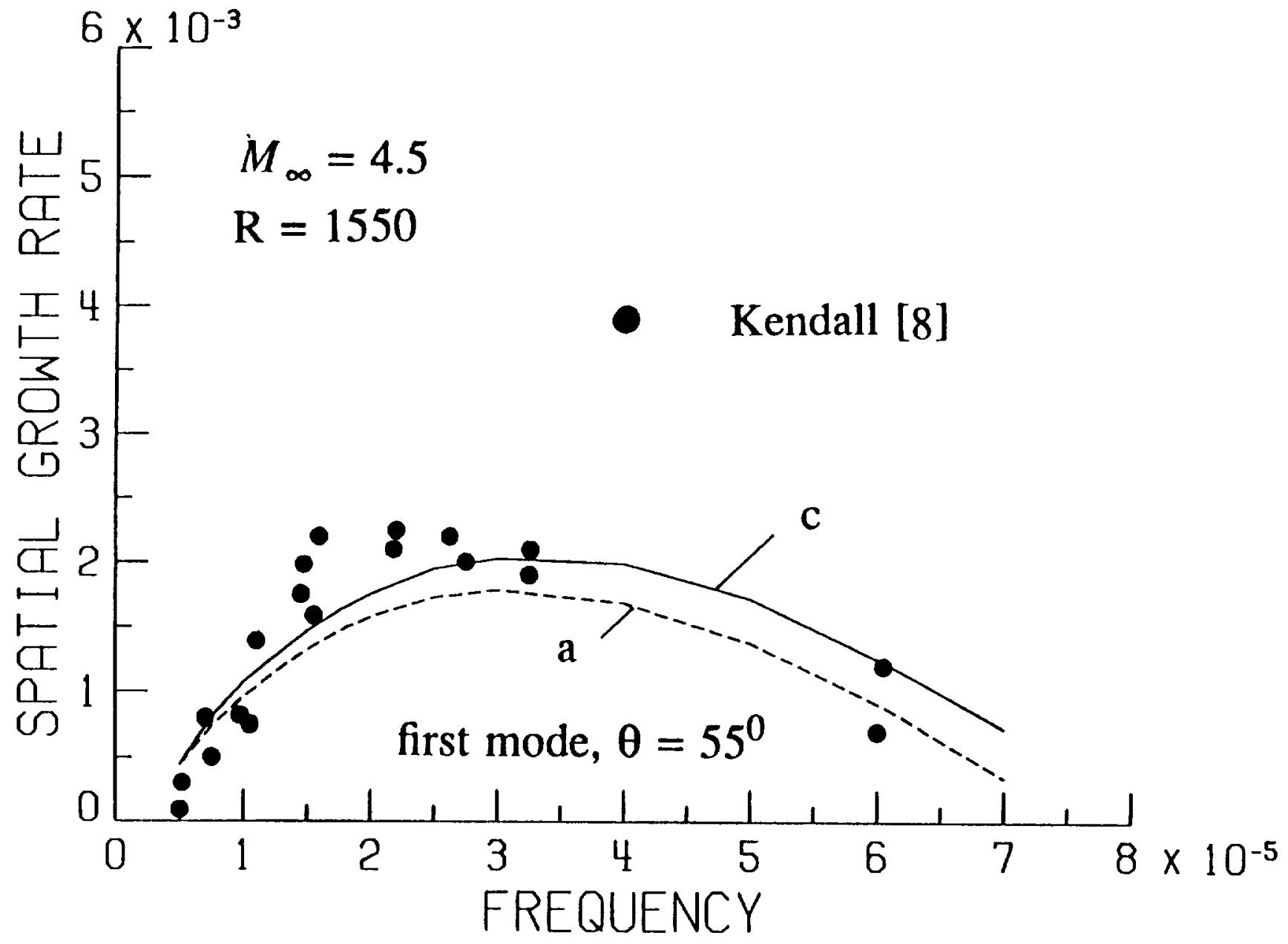

Fig 14a Comparison of the nonparallel growth rate with Kendall's experiment for an oblique first mode at $M_{\infty}=4.5, R=1550$. [a| $-\alpha_{i}$, [c] Eq 33 based on $\zeta_{9} \max$. 


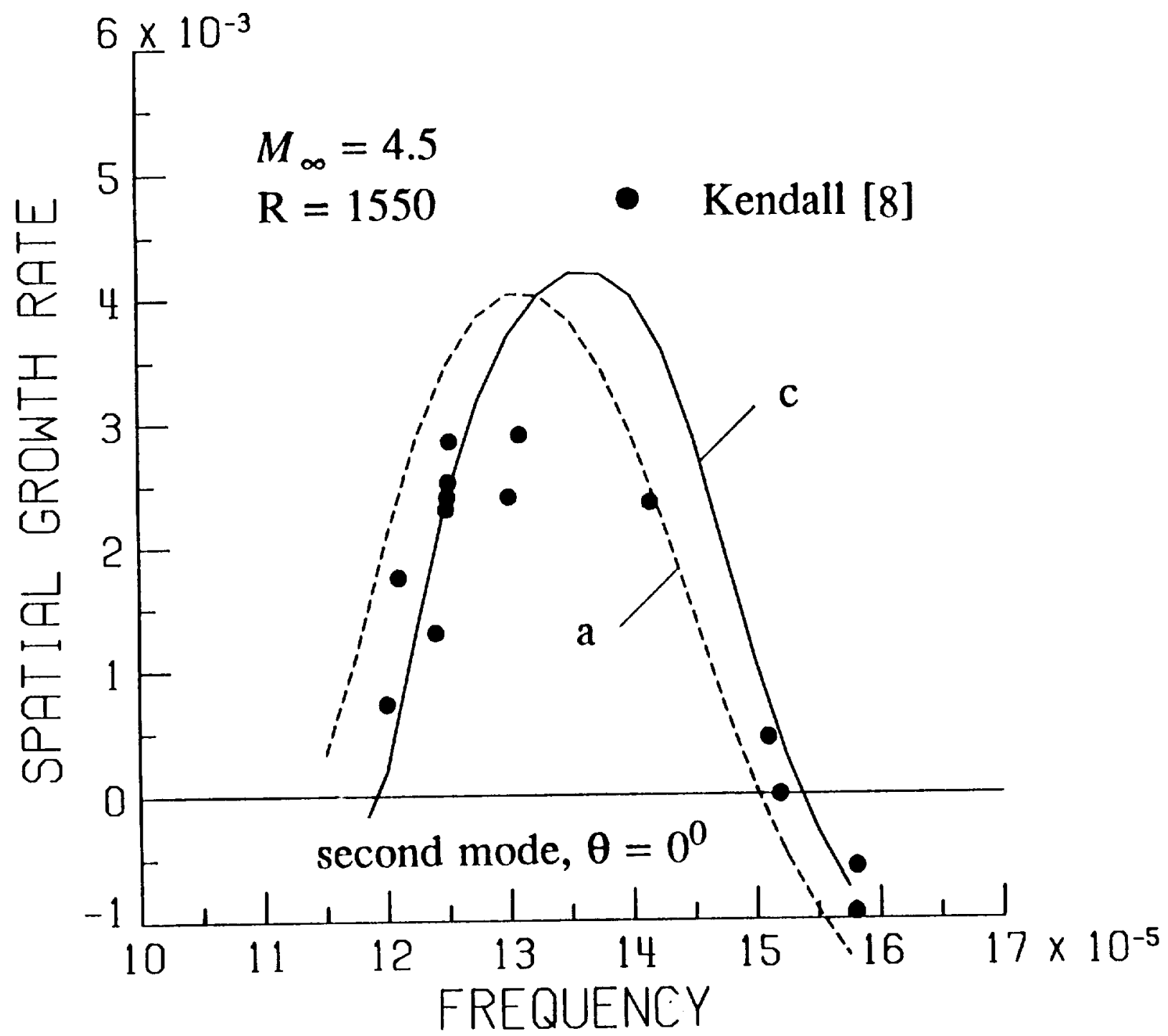

Fig 14b Comparison of the nonparallel growth rate with Kendall's experiment for a 2D second mode at $M_{-}=4.5, R=1550$. [a] $-\alpha_{i}$, [c] Eq 33 based on $\zeta_{9}$ max. 

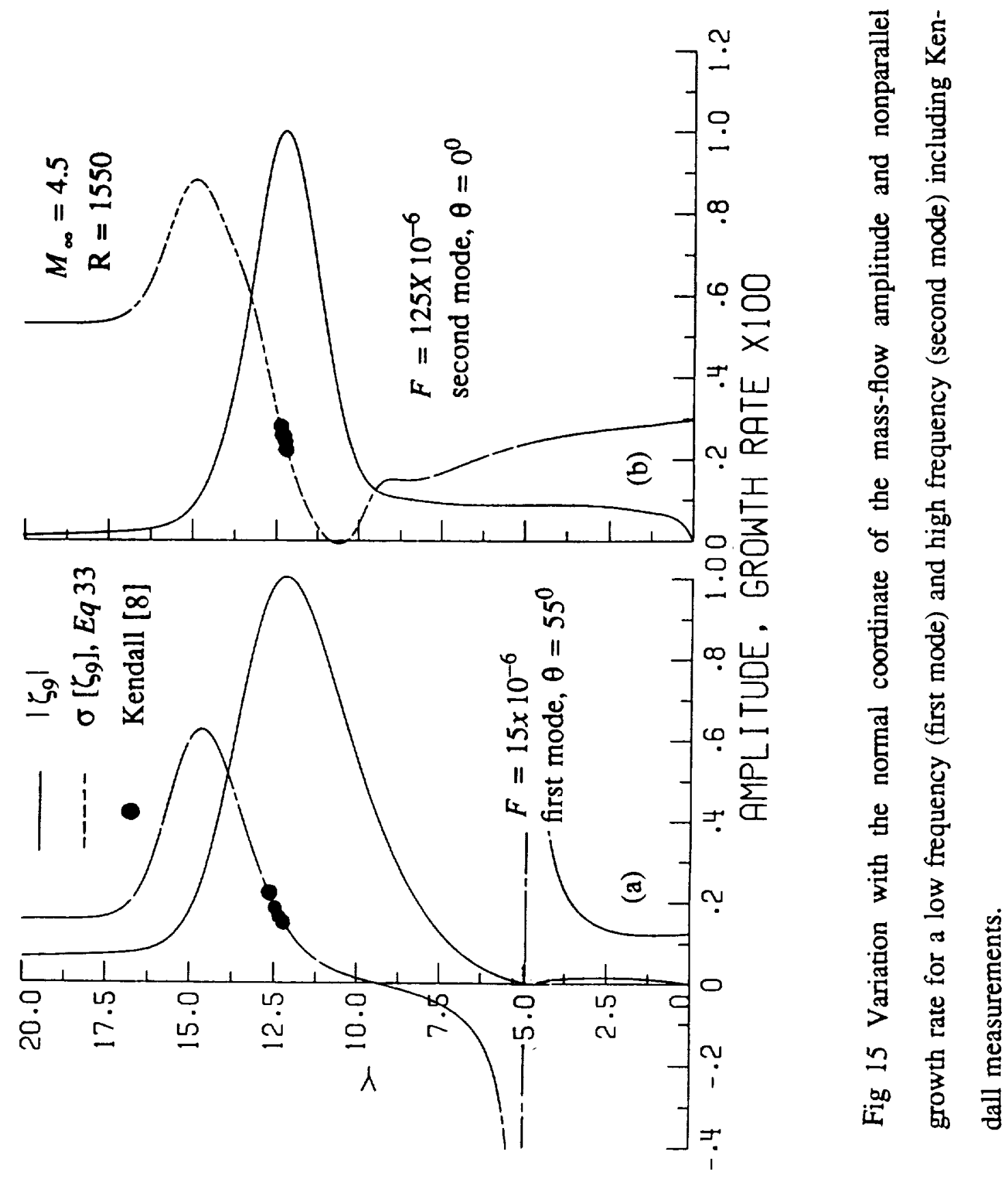


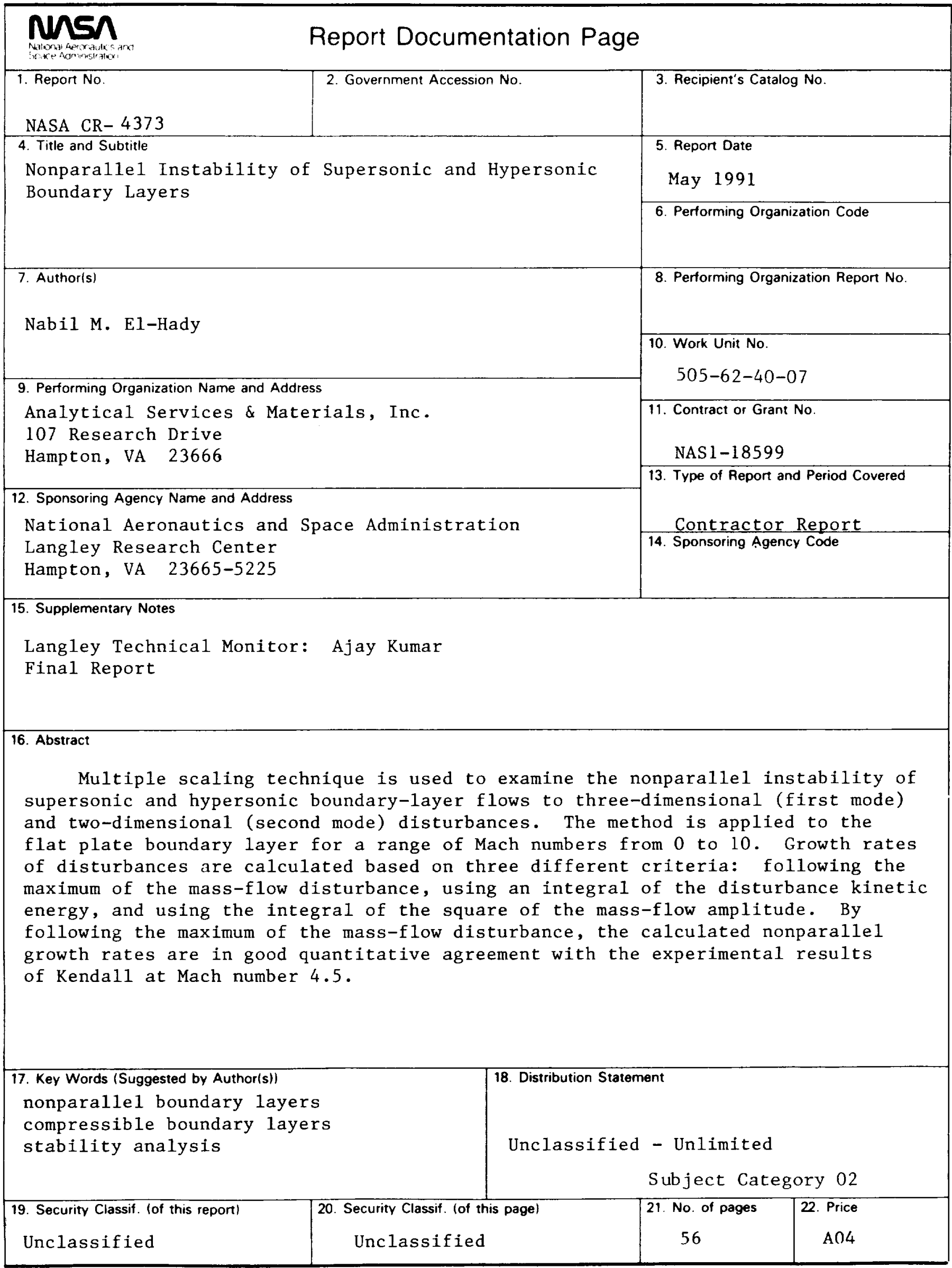





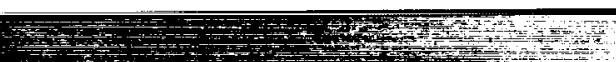

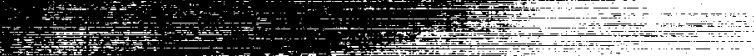

(1)

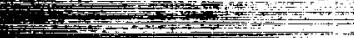

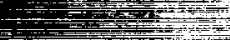

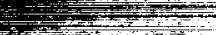

ato

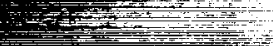

1)

ard

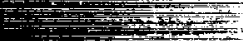

$+\frac{1}{2}$

and

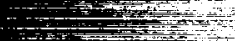

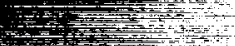

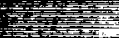

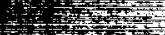

$+3 x+1+13=$

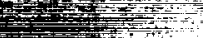

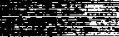

$\Rightarrow=$

$1+\frac{1}{12}$

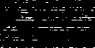

$1-3=20$

4

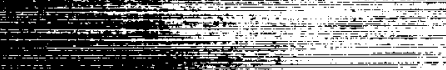

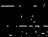

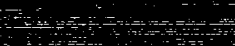

$-1,1$

$\frac{-b i x}{4}$

$\frac{1}{10}=$

$x+\frac{x}{20}$

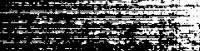

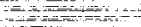

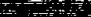

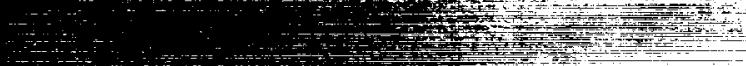

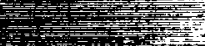

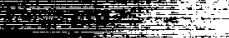

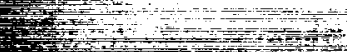

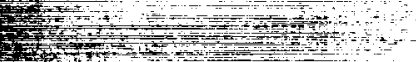

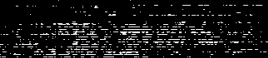

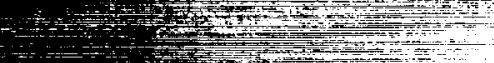

$+3 \times 1,1010$

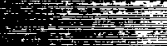

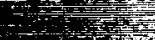

-

The $=0$

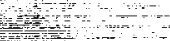

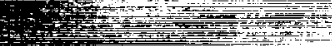

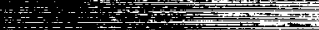

1.

D

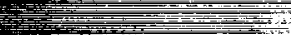

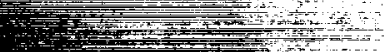

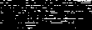

$2=1$

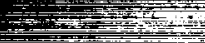

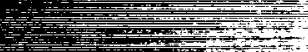

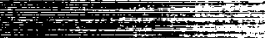

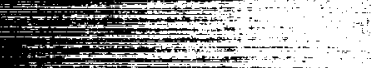

$+2=$

$\pm \pm x+1$

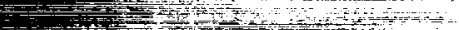

$=-1.10$

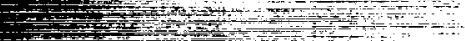

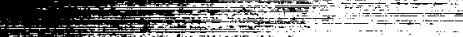

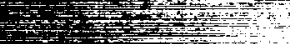

initis

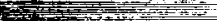

7)

1) 1 (1)

$\sin 2$

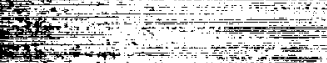

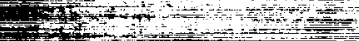

$3+14+2+1,4$

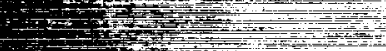

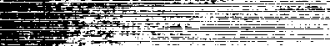



$3+3$

$-1$

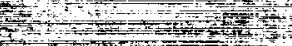

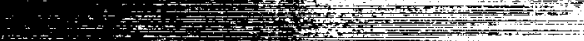

(1)

$\rightarrow+$

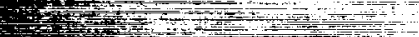

11

$-1$

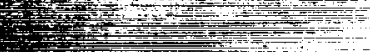


